\title{
MOD-DR: Microgrid Optimal Dispatch with Demand Response
}

\author{
Ming Jin ${ }^{\mathrm{a}, \mathrm{b}}$, Wei Feng ${ }^{\mathrm{b}}$, Ping Liu ${ }^{\mathrm{b}}$, Chris Marnay $^{\mathrm{b}}$, Costas Spanos ${ }^{\mathrm{a}}$ \\ ${ }^{a}$ Electrical Engineering and Computer Sciences Department, University of California, \\ Berkeley, Berkeley, CA 94720, USA \\ ${ }^{b}$ Energy Technologies Area, Lawrence Berkeley National Laboratory, 1 Cyclotron Road, \\ Berkeley, CA 94720, USA
}

\begin{abstract}
In the face of unprecedented challenges of upcoming fossil fuel shortage and reliability and security of the grid, there is an increasing interest in adopting distributed, renewable, energy resources, such as microgrids (MGs), and engaging flexible electric loads in power system operations to potentially drive a paradigm shift in energy production and consumption patterns. Prior work on MG dispatch has leveraged decentralized technologies like combined heat and power (CHP) and heat pumps to promote efficiency and economic gains; however, the flexibility of demand has yet to be fully exploited in cooperation with the grid to offer added benefits and ancillary services. The object of the study is to develop microgrid optimal dispatch with demand response (MOD-DR), which fills in the gap by coordinating both the demand and supply sides in a renewable-integrated, storage-augmented, DR-enabled MG to achieve economically viable and system-wide resilient solutions. The key contribution of this paper is the formulation of a multi-objective optimization with prevailing constraints and utility trade-off based on the model of a large-scale MG with flexible loads, which leads to the derivation of strategies that incorporate uncertainty in scheduling. Evaluation using real datasets is conducted to analyze the uncertainty effects and demand response potentials, demonstrating in a campus
\end{abstract}

Email addresses: jinming@berkeley.edu (Ming Jin), WeiFeng@lbl.gov (Wei Feng), PingLiu@lbl.gov (Ping Liu), ChrisMarnay@lbl.gov (Chris Marnay), spanos@berkeley.edu (Costas Spanos)

Preprint submitted to Applied Energy

November 23, 2016

(C) 2016. This manuscript version is made available under the Elsevier user license http://www.elsevier.com/open-access/userlicense/1.0/ 
prototype a $17.5 \%$ peak load reduction and $8.8 \%$ cost savings for MOD-DR compared to the non-trivial baseline, which is on par with the Oracle for perfect predictions.

Keywords: microgrid dispatch, demand response, uncertainty, unit commitment, distributed energy resources

\section{Introduction}

The convergence and mutual strengthening of distributed generation (DG), storage, demand response, and the massive integration of sensing, communication, and control technology initiates profound changes in the energy production

5 and consumption patterns, and opens up ample opportunities for addressing the issues of upcoming fossil fuel shortages, environmental preservation, and grid reliability and security [1, 2, 3, 4. The synergistic potential, nevertheless, is yet to be fulfilled by taking up challenges like the utilization of highly intermittent renewables [2], the operation of DG in tandem with storage [5], and the transformation of domestic customers from static consumers into active participants [6.

The envisioned solutions are multifaceted, but from the practitioners' point of view, they can be oriented towards the supply or demand sides. The division of the grid into productive sub-systems - so-called microgrids (MGs), which integrate DG and storage for local demand - has been proposed to increase manageability and reduce transportation losses [7, 8, 9. A MG can be either connected to other MGs and/or the main grid for energy exchange, or run in island mode as circumstances or economics dictate [3, 9, 10, 11, 8]. The efficiency and environmental benefits also arise from the adoption of combined heat and 20 power (CHP) by reusing the generator's waste heat to simultaneously provide electrical and thermal energy [9, 12, 13. On the other hand, active demand response (DR), defined as "changes in electric usage implemented directly or indirectly by end-use customers / prosumers from their current consumption / injection patterns in response to certain signals" [6], has been put forward 
and implemented to lower peak energy usage and utility bills, and, at the same time, bring higher capacity factor and security of distribution grids [14, 15]. The paradigm shift can be further driven by the offer of incentive-based DR contracts [6, 16, and the institution of time-differentiated pricing, e.g., timeof-use (TOU) and real-time pricing (RTP), which reflect fluctuating wholesale 30 prices to the end users and encourage responsive load shifting and curtailment [3.

While the flexibility and value brought by DR to energy systems have been widely recognized, they have not been fully captured in the operation of MG. Despite the challenges of a comprehensive treatment of DR together with the uncertainty inherent in renewables and wholesale market prices, the potential social and economic impacts are substantial and worthwhile of further investigation [6, 2, 1].

It is, therefore, the object of the study to develop microgrid optimal dispatch with demand response (MOD-DR), which fills in the gap by simultaneously 40 exploiting both the demand and supply sides in a renewable-integrated, storageaugmented, DR-enabled MG to achieve economically viable and system-wide resilient operational solutions.

The rest of the paper is organized as follows. Previous works are surveyed in Section 2, with an emphasis on modeling, and dispatch and DR under uncer45 tainty. Section 3 discusses the modeling of MG with flexible demands, including the problem formulation, the MG coordinator and DR mechanism, typical DG technologies and load profiles of buildings connected to a pipeline network. Section 4 deals with planning under uncertainty, which includes the forecasting methodology, and the day-ahead and adaptive dispatch strategies. The dataset and implementation are discussed in Section 5 , followed by cost analysis (Section 6) and a case study for the day-ahead and adaptive strategies in a practically-oriented setting (Section 7). Conclusions are drawn in Section 8. 


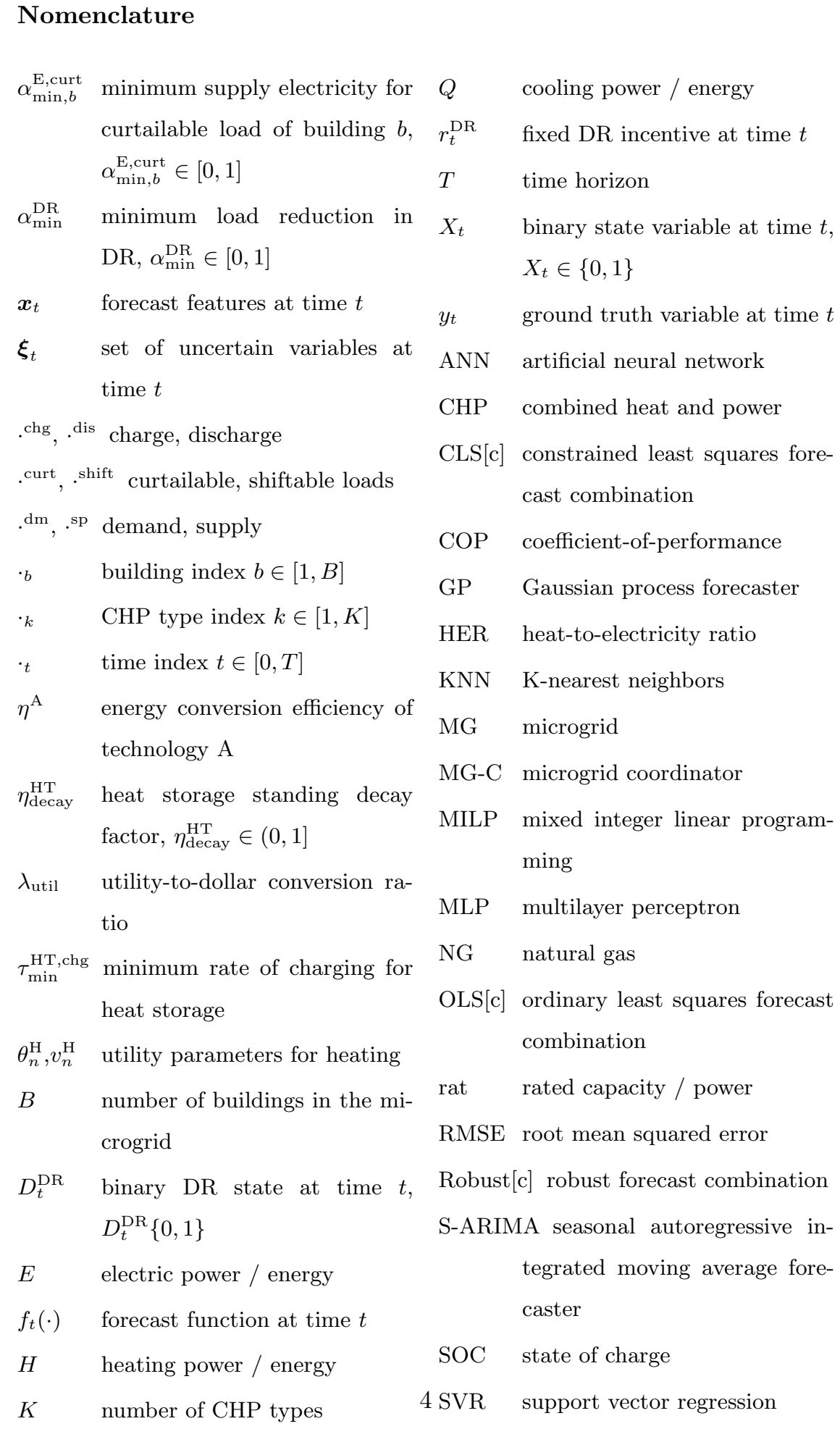




\section{Related Work}

Previous work has been undertaken on modeling high-level system design

55 5, 18, 13, 9. The average long-run operational and real options valuation have been incorporated in [19, 18, 13, to examine the economics of MG with a diverse combination of CHP, boilers, chillers, electric batteries, thermal storage, solar panels, and wind turbines. Though this work lent insight into the modeling of price and renewables, becomes a disadvantage for daily operational planning.

The dispatch of MG is, in essence, a unit commitment (UC) problem, which refers to optimizing generation resources over a short time horizon to satisfy load demand at minimum operational cost while satisfying prevailing constraints (LP) 21, mixed integer linear programming (MILP) [22, 10, 23, dynamic programming [24, 12, 13], simulated annealing [3, artificial neural networks [25], particle swarm optimization [18, hybrid methods [26] and evolutionary algorithms [27, 5], as well as game theoretic agent-based formulations [7]. Mohamed nance cost and emission reduction of a MG based on game theory. Chaouachi et al. 21 proposed a MG energy management approach using LP with predicted renewable energy resources and load demand. Kriett and Salani 23] targeted a residential MG with energy storage for operating cost minimization using MILP, namic programming (DP) has been employed by Kitapbayev et al. [12] and Díaz and Moreno [13] to explore different modes of operation to maximize the total payoff, yet DP relies on the discretization of states and is susceptible to computational and memory limitations. Ommen et al. 29] conducted an empirical comparison of LP, MILP, and non-linear programming (NLP), and concluded that MILP is most appropriate from the viewpoint of accuracy and runtime, with the added capability of dealing with the part-load region as in CHP. In 
light of their findings, and the growing interest in MILP driven by the enhancement of the performance of MILP solvers such as CPLEX and Gurobi [30, 20], we leverage the accuracy and scalability of MILP in our formulation, with the added benefit of enabling the incorporation of DR that has been missing in previous MG operation studies.

The uncertainty that arises from penetration of renewables and volatility of pool prices also exerts an impact on MG dispatch. Also, due to the intermittent renewable generation and the limit of power transmission and distribution systems, renewables are often curtailed in many places of the world [31; thus, it makes DR more useful by helping renewables be locally consumed. The effects of forecasting errors on the economic value of dispatch have been analyzed for the prediction of electricity price [22, 32, loads [33, 34, and weather [10], and there is consensus that the optimality of dispatch and the accuracy of forecasts are concomitant. Uncertain variables can be systematically considered in dispatch planning through prediction [1, 35, 36, 37, 33] or stochastic programming (SP) 38, 5, 18. Park et al. 36] studied a UC algorithm anticipating a given load-uncertainty. Delarue and D'haeseleer [33] developed an adaptive MILP UC strategy to determine the value of forecasting. Compared to the present study, they have more simplistic assumptions on load and generators. Baziar and Kavousi [18] employed the $2 \mathrm{~m}$ point estimate method, which conducts $2 \mathrm{~m}$ runs of the deterministic framework for each of the $m$ uncertain variables in terms of the first three moments of the relevant probability density function, and the optimal solutions are aggregated to find the expected value of the objective function. Morales et al. 38] and Niknan et al. [5] account for stochasticity by generating a large number of scenarios, i.e., realizations of the random variables throughout the horizon, and aggregate the solutions to obtain the dispatch plan. The main concerns about SP include the reliability of the distribution approximation to the true underlying process, and the scalability and computational issues that arise from the augmented problems. In terms of methodology, we find similarity with the work by Molderink et al. [1], who proposed the three-step control methodology of prediction, planning, and real-time control, though their 
approach is iterative dynamic programming with agent-based load scheduling,

\section{The Model of MG with Flexible Demand}

The problem of optimal MG dispatch with DR is formulated within an optimization framework. The models of the key components, including the MG coordinator, building loads, and generator and storage technologies, are elabo- 


\subsection{Problem Formulation}

The key problem to tackle is: "How do we coordinate load and generation under demand response on a district energy level to ensure economic viability of the microgrid and system-wide resilience?", where two prominent factors are involved:

- Elasticity of loads for individual buildings under the MG management

- Uncertainty and fluctuation of energy demands, electricity tariffs, and weather conditions

The elasticity aspect, crucial yet under-exploited in previous work, can unlock the potential of substantial peak demand reductions through either load shaving or shifting, which can benefit the MG by lowering the energy bill, and the grid in the form of ancillary services. It entails a trade-off among the operation costs, the utility (i.e., the satisfaction derived from energy consumption) for each building, and the DR incentives offered by the grid. Section 3 introduces the modeling of both the supply and demand sides of the MG as is shown in Fig. 1. The uncertainty part, inherent for all dispatch planners, is dealt with by the forecasting module of MG-Coordinator (MG-C); several methods are presented in Section 4.1 .

The basic optimal dispatch problem can be formulated by:

$$
\begin{aligned}
& \min _{\left\{\boldsymbol{x}_{t}\right\}_{t=1}^{T}} \sum_{t=1}^{T} f_{t}^{\mathrm{Ope}}\left(\boldsymbol{x}_{t}, \boldsymbol{\xi}_{t}\right)+f_{t}^{\mathrm{Mtn}}\left(\boldsymbol{x}_{t}, \boldsymbol{z}_{t}, \boldsymbol{\xi}_{t}\right)-R_{t}^{\mathrm{DR}}-\lambda_{\mathrm{util}} u_{t}\left(\boldsymbol{x}_{t}\right) \\
& \text { s.t. } \quad \boldsymbol{x}_{t} \in \mathcal{X}_{t}\left(\boldsymbol{z}_{t}, \boldsymbol{\xi}_{t}\right), \boldsymbol{z}_{t} \in \mathcal{Z}_{t}\left(\boldsymbol{z}_{t-1}, \boldsymbol{x}_{t}\right), \forall t=1, \ldots, T
\end{aligned}
$$

where $\boldsymbol{x}_{t}$ is the dispatch proposal at time $t$, which includes variables within four categories: generation, e.g., power from CHP and PV; storage, e.g., charging/discharging with the cooling storage; grid import/export; and supply for buildings. The state variable, $\boldsymbol{z}_{t}$, captures the state-of-charge (SOC) of the storage as governed by the previous state and action: $\boldsymbol{z}_{t}=\phi\left(\boldsymbol{z}_{t-1}, \boldsymbol{x}_{t}\right)$. The uncertain quantities, e.g., solar irradiation $\operatorname{Irr}_{t}$ and electricity price $c_{t}^{\text {grid }}$, are summarized in $\boldsymbol{\xi}_{t}$. 


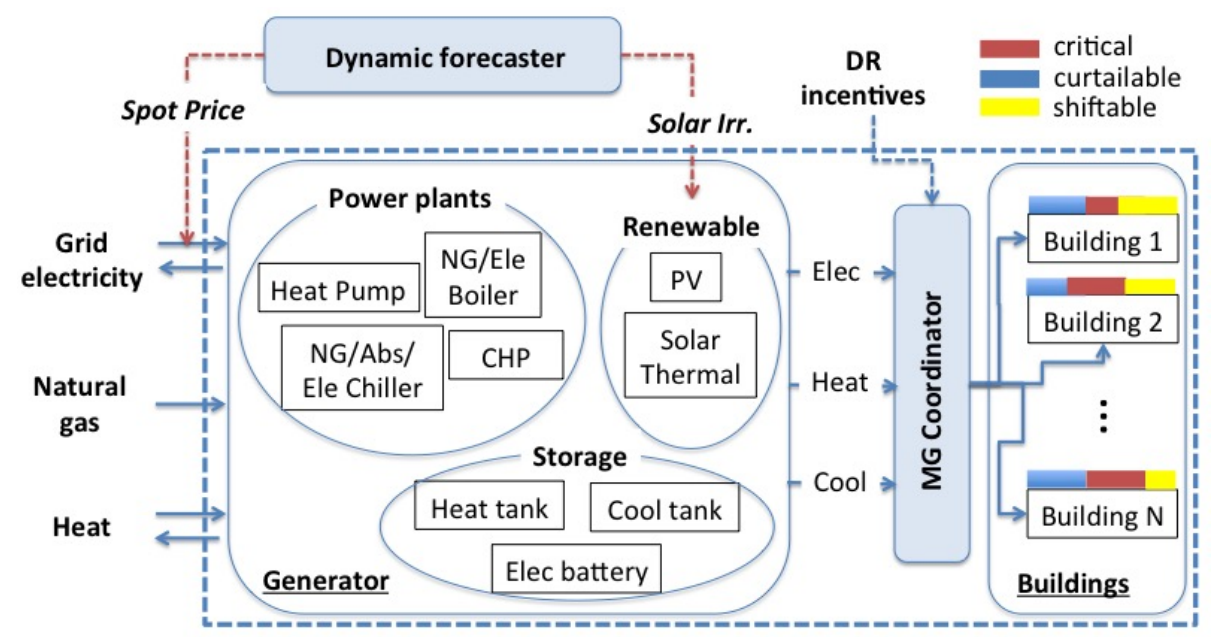

Figure 1: System overview. The MG owns a generation facility, and can exchange energy with the grid to meet the building demands. Additionally, the uncertain variables and DR incentives are incorporated during planning.

Objective function. $f_{t}^{\mathrm{Ope}}\left(\boldsymbol{x}_{t}, \boldsymbol{\xi}_{t}\right)$ is the expenditure on electricity, heat, and gas to operate the generation facility, net of any electricity or heat sold back. The maintenance cost $f_{t}^{\mathrm{Mtn}}\left(\boldsymbol{x}_{t}, \boldsymbol{z}_{t}, \boldsymbol{\xi}_{t}\right)$ refers to the expense for facilities with on-site personnel; it is proportional to the amount of power that has been generated. Natural gas-fueled devices are usually more costly to maintain than their electric counterparts; the marginal price also decreases as capacity expands.

On top of the former commonly adopted conditions [12, 29, 10, the DR incentive, $R_{t}^{\mathrm{DR}}$, and building utility, $u_{t}\left(\boldsymbol{x}_{t}\right)$, are incorporated in order to reveal the effect of demand response. MG-C, as a result, is able to offer guidance for load shaping based on individual building profiles, as enforced by financial contracts or service agreements, where $\lambda_{\text {util }} \geq 0$ acts as the trade-off parameter for building satisfaction.

Building utility. As the MG-C assesses the potential dispatch strategies, individual buildings are able to manifest their propensity to consume by the specification of: 
- Demand profiles, consisting of critical, curtailable, and shiftable loads

- Utility functions to express the rigidity with respect to load shaving and shifting

While the former indicates the amount of loads that are DR-enabled / disabled, the latter depicts a continuum of satisfaction mappings, generally employed in game-theoretic scheduling [46] and agent-based controls [44, 14]. In particular, $u_{t}\left(\boldsymbol{x}_{t}\right)$ comprises of utility functions for curtailable and shiftable loads, which are piece-wise linear, non-decreasing, and concave,

$$
\begin{gathered}
u_{t}\left(\boldsymbol{x}_{t}\right)=\sum_{b \leq B} u_{b}^{\mathrm{E}, \mathrm{shift}}\left(\left\{E_{t^{\prime}, b}^{\mathrm{sp}, \mathrm{shift}}\right\}_{t^{\prime} \leq t}\right)+u_{b}^{\mathrm{E}, \mathrm{curt}}\left(E_{t, b}^{\mathrm{sp}, \mathrm{curt}}\right) \\
+u_{b}^{\mathrm{H}}\left(H_{t, b}^{\mathrm{sp}}\right)+u_{b}^{\mathrm{Q}}\left(Q_{t, b}^{\mathrm{sp}}\right)
\end{gathered}
$$

where $E_{t, b}^{\mathrm{sp}, \mathrm{shift}}$ and $E_{t, b}^{\mathrm{sp}, \mathrm{curt}}$ represent the shiftable and curtailable loads supplied at time $t$ for building $b$, and $H_{t, b}^{\mathrm{sp}}$ and $Q_{t, b}^{\mathrm{sp}}$ denote the heating and cooling supply.

The shiftable load utility $u_{b}^{\mathrm{E}, \mathrm{shift}}\left(\left\{E_{t^{\prime}, b}^{\mathrm{sp}, \text { shift }}\right\}_{t^{\prime} \leq t}\right)$ promotes early completion of shiftable tasks and penalizes deferred loads. We define $\bar{p}_{t, b}^{\mathrm{E}, \mathrm{shift}}=\frac{\sum_{t^{\prime} \leq t} E_{t, b}^{\mathrm{sp}, \mathrm{shift}}}{\sum_{t^{\prime} \leq T} E_{t, b}^{\mathrm{dm}, \mathrm{shift}}}$ as the percentage of total shiftable demands satisfied at time $t$; then $u_{b}^{\mathrm{E}, \text { shift }}\left(\left\{E_{t^{\prime}, b}^{\mathrm{sp}, \mathrm{shift}}\right\}_{t^{\prime} \leq t}\right)$ is piece-wise linear with decreasing slopes 1 Intuitively, the no-complaint scenario unfolds when all the jobs up to the current time are completed, while the diminishing marginal return brings flexibility to load shifting.

The curtailable utility, $u_{b}^{\mathrm{E}, \mathrm{curt}}\left(E_{t, b}^{\mathrm{sp}, \mathrm{curt}}\right), u_{b}^{\mathrm{H}}\left(H_{t, b}^{\mathrm{sp}}\right)$, and $u_{b}^{\mathrm{Q}}\left(Q_{t, b}^{\mathrm{sp}}\right)$, share the property of discouraging unfulfilled demands at any time. For heating, as an example, the proportion of heat demand satisfied is $\bar{p}_{t, b}^{\mathrm{H}}=\frac{H_{t, b}^{\mathrm{sp}}-\alpha_{\min , \mathrm{b}}^{H} H_{t, b}^{\mathrm{dm}}}{\left(1-\alpha_{\min , \mathrm{b}}^{H}\right) H_{t, b}^{\mathrm{dm}}} \in$

\footnotetext{
${ }^{1}$ The value of $u_{b}^{\text {E,shift }}\left(\left\{E_{t^{\prime}, b}^{\text {sp,shift }}\right\}_{t^{\prime} \leq t}\right)$, which has slopes $v_{n}^{\text {E,shift }}$ on segments $\left[\theta_{n-1}^{\mathrm{E} \text {,shift }}, \theta_{n}^{\mathrm{E} \text {,shift }}\right]$, with $v_{1}^{\mathrm{E} \text {,shift }} \geq \cdots \geq v_{N^{\mathrm{E}, \text { shift }}}^{\mathrm{E} \text {,sift }} \geq 0$, and $0=\theta_{0}^{\mathrm{E} \text {,shift }}<\cdots<\theta_{N^{\mathrm{E}, \text { shift }}}^{\mathrm{E} \text {, } h \text { ift }}=1$, is given by the linear program (LP):

$$
u_{b}^{\mathrm{E}, \text { shift }}\left(\left\{E_{t^{\prime}, b}^{\mathrm{sp}, \text { shift }}\right\}_{t^{\prime} \leq t}\right)=\max _{\left\{\psi_{n}\right\}_{n=1}^{N \text { E,shift }}} \sum_{n=1}^{N^{\mathrm{E}, \text { shift }}} v_{n}^{\mathrm{E}, \text { shift }} \psi_{n}
$$

where $0 \leq \psi_{n} \leq \theta_{n}^{\mathrm{E} \text {,shift }}-\theta_{n-1}^{\mathrm{E} \text {,shift }}$ for $n=1, \ldots, N^{\mathrm{E} \text {,shift }}$, and $\sum_{n=1}^{N^{\mathrm{E}, \text { shift }}} \psi_{n}=\bar{p}_{t, b}^{\mathrm{E} \text {,shift }}$.
} 

therefore, is regarded as the utility-to-dollar conversion ratio, in the light of the multi-objective formulation that tailors the treatment of $\underline{\mathrm{P} 0}$ with varying DR commitment.

Power constraints and system resilience. Above all, it is crucial to maintain the balance between load and generation at all times [47, 5, 10]. For electricity (and heat), we enable purchasing / selling electricity (and heat) from / to the grid (industry heat network) (see Appendix .1 for thermal balances),

$$
\begin{aligned}
& \left(E_{t}^{\text {grid,pur }}-E_{t}^{\text {grid,sal }}\right)+\sum_{k \leq K} E_{t, k}^{\mathrm{CHP}}+E_{t}^{\mathrm{PV}}+\left(E_{t}^{\text {Bat,from }}-E_{t}^{\text {Bat,sto }}\right) \\
& =\sum_{\text {tech } \in \mathcal{S}_{E, \mathrm{dm}}} E_{t}^{\mathrm{tech}}+\sum_{b \leq B}\left(E_{t, b}^{\mathrm{sp}, \text { shift }}+E_{t, b}^{\mathrm{sp}, \text { critic }}+E_{t, b}^{\mathrm{sp}, \text { curt }}\right)
\end{aligned}
$$

where $\mathcal{S}_{E, \mathrm{dm}}$ is the set of technologies that consume electricity, including the electric chiller and boiler, and heat pump.

The dispatch variables are confined to the feasible set $\mathcal{X}_{t}\left(\boldsymbol{z}_{t}, \boldsymbol{\xi}_{t}\right)$ delineated by the power balances, the generation and storage technologies, e.g., CHP partial loads, PV outputs, and charge/ discharge rate limit for storage, DR and load stipulations (Section 3). The states $\boldsymbol{z}_{t}$ are constrained to $\mathcal{Z}_{t}\left(\boldsymbol{z}_{t-1}, \boldsymbol{x}_{t}\right)$ defined by the dynamic charging behaviors and the capacity limits. System resilience can be prescribed in either the cap on the total import power from the grid [44, 47, 5, 10, or the spinning reserve limits on the storage resources [47, 5].

The MG can exchange energy with the grid and dispatch generators to meet the demands of buildings, as operated through the MG coordinator (Fig. 2).

\footnotetext{
${ }^{2}$ The function value of $u_{b}^{\mathrm{H}}\left(H_{t, b}^{\mathrm{sp}}\right)$ with marginal utility $v_{n}^{\mathrm{H}}$ on segments $\left[\theta_{n-1}^{\mathrm{H}}, \theta_{n}^{\mathrm{H}}\right]$ with $v_{1}^{\mathrm{H}} \geq \cdots \geq v_{N}^{\mathrm{H}} \geq 0$, and $0=\theta_{0}^{\mathrm{H}}<\cdots<\theta_{N^{\mathrm{H}}}^{\mathrm{H}}=1$ is given by the LP:

$$
u_{b}^{\mathrm{H}}\left(H_{t, b}^{\mathrm{sp}}\right)=\max _{\left\{\psi_{n}\right\}_{n=1}^{N}} \sum_{n=1}^{N^{\mathrm{H}}} v_{n}^{\mathrm{H}} \psi_{n}
$$

where $0 \leq \psi_{n} \leq \theta_{n}^{\mathrm{H}}-\theta_{n-1}^{\mathrm{H}}$ for $n=1, \ldots, N^{\mathrm{H}}$, and $\sum_{n=1}^{N^{\mathrm{H}}} \psi_{n}=\bar{p}_{t, b}^{\mathrm{H}}$.
} 


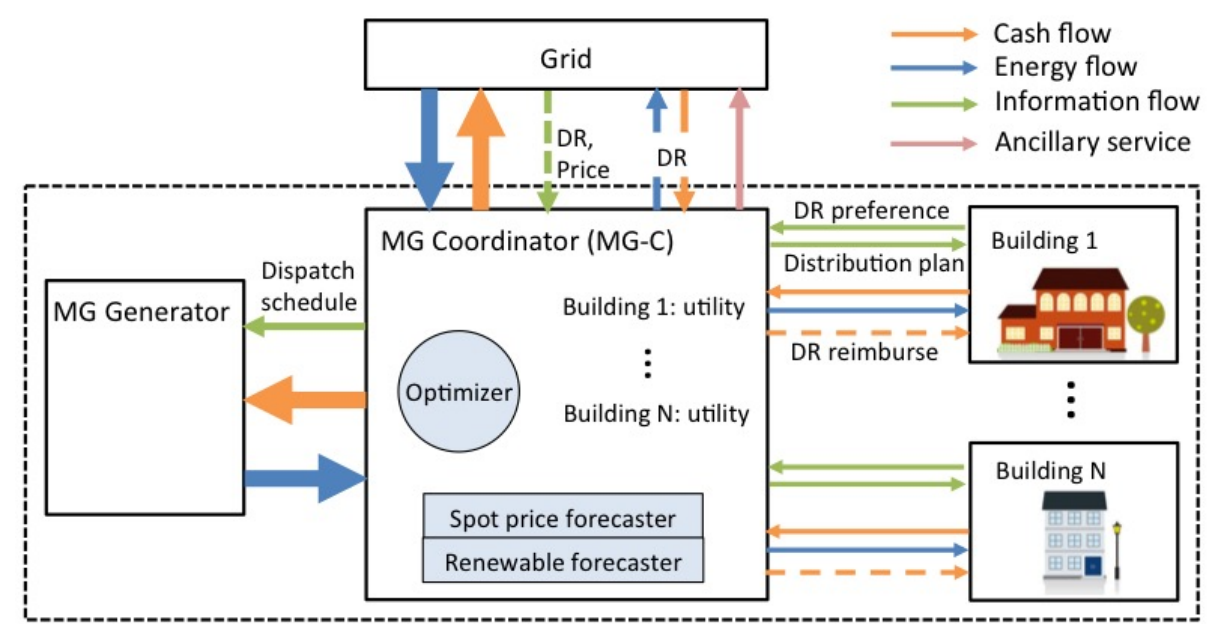

Figure 2: Illustration of the flow of payment, energy, and information among buildings, MG coordinator, and the grid under DR.

\subsection{The $M G$ Coordinator and $D R$}

The MG coordinator (MG-C) is a software-defined entity that serves the MG by managing the energy balance between demand and supply, and the risks of exposure to spot market volatility. For the particular MG under study, like university campuses [48, where the main object is energy provision rather than retailing, the MG-C is concerned about the operating costs of its generation facility and the expenditure on gas and electricity in the wholesale market, as illustrated in Fig. 2.

Apart from billing the MG on the actual electricity consumption, the network operator can provide DR signals based on market mechanisms [49, 16], $D_{t}^{\mathrm{DR}} \in$ $\{0,1\}$, up to 24 -hour ahead notice for participants to consider reducing peak demands. For MG-C, the decision of engagement, $X_{t}^{\text {DR }} \in\{0,1\}$, depends on the predicted high electricity price events, on top of the incentive contracts agreed upon with the grid. To be more specific, the MG-C will be rewarded with a fixed amount, $r_{t}^{\mathrm{DR}} \geq 0$, for partaking in DR (and potentially offering 
ancillary services), i.e., $D_{t}^{\mathrm{DR}}=X_{t}^{\mathrm{DR}}=1$ :

$$
R_{t}^{\mathrm{DR}}=r_{t}^{\mathrm{DR}} D_{t}^{\mathrm{DR}} X_{t}^{\mathrm{DR}}
$$

with the minimum requirement of $\alpha_{\min }^{\mathrm{DR}}$-percentage building load shedding 3

$$
\begin{aligned}
X_{t}^{\mathrm{DR}} & \sum_{b \leq B}\left(E_{t, b}^{\mathrm{sp}, \text { curt }}+E_{t, b}^{\mathrm{sp}, \text { shift }}\right) \\
& \leq \sum_{b \leq B}\left(1-\alpha_{\mathrm{min}}^{\mathrm{DR}}\right)\left(E_{t, b}^{\mathrm{dm}, \text { curt }}+E_{t, b}^{\mathrm{dm}, \text { shift }}\right)
\end{aligned}
$$

which is estimated in accordance with the utility's baseline methodology [14, 16].

The involvement of individual buildings in DR is voluntary, with the extent of commitment delineated by the diverse load profiles and the utility functions (1). As part of the electricity agreement, they can be encouraged to take part in DR through incentive reimbursement. Generation is dispatched based on demand, which is set depending on the predicted pool price facilitated by the MG-C, as elucidated in Fig. 2 on information flow.

\subsection{Building Loads}

The community of buildings a MG serves, including residential and commercial buildings, hospitals, and public service stations, exhibit various load profiles for heating, cooling, and electricity, as depicted in Fig. 5. Three salient types of loads are considered to facilitate planning.

Critical load. For electricity usage in data centers and ICUs of hospitals, for example, it is of utmost importance that the loads are satisfied, i.e.,

$$
E_{t, b}^{\mathrm{sp}, \text { critic }}=E_{t, b}^{\mathrm{dm}, \mathrm{critic}}
$$

\footnotetext{
${ }^{3}$ We use the big-M method to convert the constraint to the equivalent linear form:

$$
\sum_{b \leq B}\left(E_{t, b}^{\mathrm{sp}, \mathrm{curt}}+E_{t, b}^{\mathrm{sp}, \mathrm{shift}}\right)
$$$$
\leq M_{t}\left(1-X_{t}^{\mathrm{DR}}\right)+\left(1-\alpha_{\mathrm{min}}^{\mathrm{DR}}\right)\left(E_{t, b}^{\mathrm{dm}, \text { curt }}+E_{t, b}^{\mathrm{dm}, \text { shift }}\right)
$$

where $M_{t}$ can be set with a large value, e.g., $B \cdot E^{\max }+\sum_{b \leq B} E_{t, b}^{\mathrm{dm}, \text { curt }}$.
} 
used to denote supply and demand. Throughout the paper we use $t$ to denote the time step at hourly resolution.

Curtailable load. Demands of this type, such as ventilation and lighting, have limited ability to be deferred to a later time, but can be traded off with users' satisfaction in the case of demand response (DR) commitment, i.e., $X_{t}^{\mathrm{DR}}=1$ :

$$
\begin{array}{r}
\left(1-X_{t}^{\mathrm{DR}}\right)\left(1-\alpha_{\min , b}^{\mathrm{E}, \mathrm{curt}}\right) E_{t, b}^{\mathrm{dm}, \text { curt }}+\alpha_{\min , b}^{\mathrm{E}, \mathrm{curt}} E_{t, b}^{\mathrm{dm}, \text { curt }} \\
\leq E_{t, b}^{\mathrm{sp}, \text { curt }} \leq E_{t, b}^{\mathrm{dm}, \text { curt }}
\end{array}
$$

where $\alpha_{\min , b}^{\mathrm{E}, \text { curt }} \in(0,1)$ identifies the minimum requirement for curtailable electricity for each building. Heating and cooling loads, $H_{t, b}^{\mathrm{dm}}, Q_{t, b}^{\mathrm{dm}}$, are considered within this category mainly because 1 ) users typically respond to DR signal by reducing the heating / cooling consumption at the current moment, without necessarily recovering it in the future, 2) even though thermal energy has been treated as thermal storage in a sub-hour time frame [40, 45], it is rare to see shifting among several hours; as the time step in the study is an hour, the DR behavior is predominantly curtailment.

Shiftable load. Loads of this type, like electric vehicle battery charging, can be shifted in time, as long as the specified amount of total energy is expanded, which is characterized by the following relations on each day:

$$
\begin{aligned}
\left(1-X_{t}^{\mathrm{DR}}\right) E_{t}^{\mathrm{dm}, \text { shift }} & \leq E_{t}^{\mathrm{sp}, \text { shift }} \leq E^{\mathrm{max}} \\
\sum_{t \leq T} E_{t}^{\mathrm{sp}, \text { shift }} & \leq \sum_{t \leq T} E_{t}^{\mathrm{dm}, \text { shift }} \quad, \forall T \in 1, \ldots, 24 \\
\sum_{t \leq 24} E_{t}^{\mathrm{sp}, \text { shift }} & =\sum_{t \leq 24} E_{t}^{\mathrm{dm}, \text { shift }}
\end{aligned}
$$

where 9 indicates that without DR commitment, i.e., $X_{t}^{\mathrm{DR}}=0$, the hourly posted shiftable load must be fully satisfied, yet with DR the supply can span a wider range from 0 to $E^{\max }$. As stipulated in 10 , we cannot satisfy shiftable loads before they occur; or rather, we can only meet demands up to the current 

be satisfied, as in (11). We do not further differentiate between interruptible and non-interruptible loads as in 44, inasmuch as our decision is made at an aggregated building scale, rather than on an individual device level.

While neglected in previous MG models [12, 29], thermal losses through pipelines are pertinent to evaluate the environmental impact and dispatch optimality [50]. We consider a pre-installed pipeline system for heat and cooling energy transfer, with the assumption that no thermal exchange exists among buildings, as illustrated in Fig. 3 for some probable topologies. Two forms of thermal losses are examined, namely, length follow and load follow modes (see

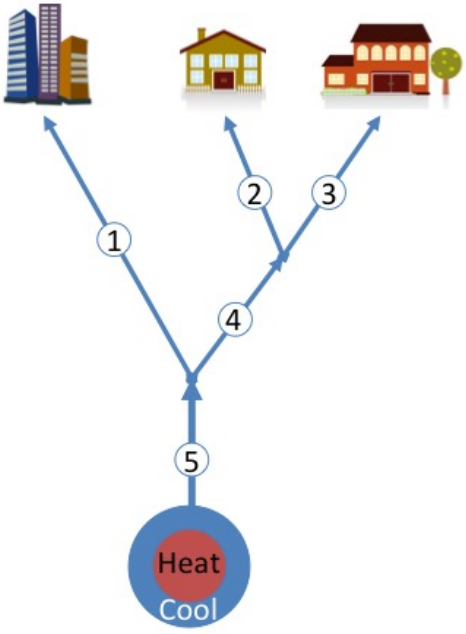

(A) Tree topology

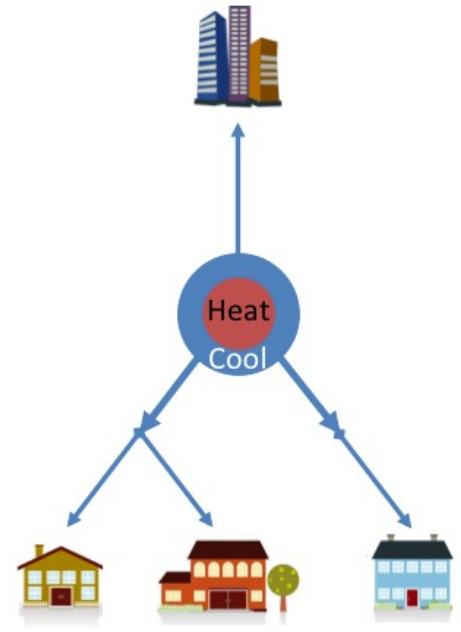

(B) Star topology

Figure 3: Illustrations of two possible topologies of pipeline network for thermal energy transportation.

\subsection{Generator and Storage Technology}

The next sections provide an overview of the technology components and requirements, with the implementation detailed in the Appendix. 
Combined Heating and Power (CHP). The coupling of power and heat production in CHP makes it an option for MGs to reduce fuel imports, lower carbon dioxide emissions and tackle fuel poverty by increasing efficiency; for instance, more than $99.7 \%$ of electricity in the Danish energy system originates from CHP and renewables [29]. The MG model allows the consideration of several CHP systems of different capacities (see Appendix .3.

65 Electric, natural gas, and absorption chillers / boilers. Heat from a liquid is removed in a chiller via a vapor-compression or absorption refrigeration cycle, where the input energy can be electricity (electric chiller), natural gas (NG chillers), or heat from steam or hot water (absorption chiller). The coefficientof-performance (COP) is typically used to depict the conversion efficiency as 270 the ratio between output cooling to input power. As for the boilers, that thermal energy is generated from natural gas combustion (NG boiler) or electric resistance heating (electric boiler).

Heat pump (HP). In a MG, a heat pump, which transports thermal energy from the source to the destination, can decouple the production constraints of the coproduced products, while maintaining high energy efficiency [51. The HP is often more efficient than its alternatives, especially in heating mode (different types of HP, such as air sourced HP, ground sourced HP, water sourced HP, differ in the COPs, which also depend on climate conditions), notwithstanding that the installed cost is significantly higher [29].

${ }_{20}$ Solar thermal and photovoltaics $(P V)$. The increasing tendency for using renewables to reduce carbon footprints has brought about widespread adoption of solar thermal and PV technology [5]. For PV, the electric power is proportional to the solar irradiation, which is often the source of volatility. Strategies introduced in Section 4 are aimed at coping with the uncertainty.

Storage Technology. Storage is conducive to smoothing variable generation and system-wide reliability. The taxonomy proposed by 52 classifies the storage 
types into sensible, latent, and thermochemical storage, of which the first category is most common because it does not involve phase change. We build a generic storage prototype for heating, cooling, and electricity with dynamic charging / discharging behaviors and standing loss, as illustrated below for the heat storage (see Appendix .4). Maintaining a minimum amount of stored energy, typically $5 \%$ of the total capacity, i.e., state-of-charge (SOC), is commonly referred to as the spinning reserve requirement [47, 5].

In summary, the modeling of various technologies enables the user to tailor the treatment of individual cases by selecting the available generators or storage. Additionally, building loads are modeled with a varying degree of flexibility to be responsive to DR. The following section discusses the coordination of the supply and demand sides to inform optimal dispatch of MG.

\section{Dispatch under Uncertainty}

MOD-DR uses state-of-the-art forecasting models to design the operation plan. It is the aim of this section to introduce two dispatch strategies, namely, day-ahead and adaptive methods, which have different emphases on the convenience of implementation or the responsiveness to unexpected changes.

\subsection{Forecasting Methodology}

The primary aim of forecasting is uncertainty reduction. For MOD-DR , accurate prediction of electricity price and solar irradiation can reduce the risks of spot market / renewable volatility through arbitrage opportunities.

Methods for solar forecasting can be grouped into time series models and numerical weather prediction (NWP) [53. Weron 54] recently conducted a comprehensive review of spot price prediction approaches, which fit into six categories: multi-agent in a game-theoretic framework, fundamental methods that incorporate physical and economic factors, reduced-form that characterizes statistical properties over time, as well as statistical approaches and computational intelligence. While reduced-form models, like the mean-reverting Orstein-

Uhlenbeck process, excel at derivatives valuation and risk analytics and have 
been employed in previous works [12, 13, 13, their simplicity and analytical tractability are no longer an advantage for prediction; in contrast, methods in the latter two classes, like autoregressive (AR)-type models, support vector regression (SVR), and artificial neural networks (ANN), can handle complex320 ity and non-linearity, and thus will be considered in this research. Additionally, forecast combinations, such as ordinary least squares (OLS[c]), constrained least squares (CLS[c]), and variance-based scheme, pointed as future directions in [54, are evaluated. These methods are also applicable to solar irradiation. For load prediction, the methods can be grouped into either data-driven methods, which resort to regression on indicators like occupancy and weather, or model-based methods, which consider the operation of building components like lighting and HVAC systems, occupancy and their behaviors [55, 56, 57, 58. Nevertheless, in this study, we assume the loads to be given, though in practice they can be predicted using the methods outlined below.

330 Baselines. The baseload price model (BPM) predicts the next day price using the median of electricity prices in the past month, due to its robustness to outliers. The 24 hourly prices of the current day can also be used, as in the Naïve model, a standard reference estimator.

Statistical approaches. The seasonal autoregressive integrated moving average (S-ARIMA) is a stochastic process coupling the AR and MA components, after differencing at appropriate time steps to remove any trends. Formally, it is given by $\operatorname{ARIMA}(p, d, q) \times(P, D, Q)_{S}$ :

$$
\underbrace{\Psi(B) \Psi_{S}\left(B^{S}\right)}_{\mathrm{AR}} \underbrace{\Delta^{d}(B) \Delta^{D}\left(B^{S}\right)}_{(\text {non-) seasonal difference }} y_{t}=\underbrace{\Phi(B) \Phi_{S}\left(B^{S}\right)}_{(\text {non-) seasonal MA }} w_{t}
$$

where $y_{t}$ is the electricity price at time $t, w_{t}$ is the white noise process, $B$ and ${ }_{335} \Delta$ are the backward shift and difference operators, i.e., $\Delta(B) y_{t}=(1-B) y_{t}=$ $y_{t}-y_{t-1}, \Psi, \Psi_{S}, \Phi, \Phi_{S}$ are polynomials of order $p, P, q, Q$ in the model specification. The seasonal lag $S=24$ is used to capture daily patterns, and the orders of MA and AR are informed by the autocorrelation and partial autocorrelation graphs. Ordinary least squares (OLS) represents linear regression-type 

tional intelligence methods, due to the exclusive reliance on historical prices, though exogenous variables like weather and power supply and demands can be integrated once available.

Computational intelligence (CI). A succinct summary of a broad category of CI methods is based on the empirical risk minimizer (ERM) framework [59]:

$$
\min _{f \in \mathcal{F}} \frac{1}{n} \sum_{i=1}^{n} l\left(f\left(\boldsymbol{x}_{i}\right), y_{i}\right)
$$

which finds a function $f \in \mathcal{F}$ of features $\boldsymbol{x}_{i}$ to minimize the loss evaluated on 345 the training set $\left\{\left(\boldsymbol{x}_{i}, y_{i}\right)\right\}_{i=1}^{n}$. It can be regarded that variants within the ERM differ by the choices of function class $\mathcal{F}$ and loss $l(\cdot, \cdot)$. Support vector regression (SVR) "lifts" the original features into higher dimensions, and optimizes $l(\cdot, \cdot)$ as the hinge loss [59]. Ridge, Lasso, and Elastic Net have been widely applied in prediction tasks and compressed sensing [59, which assume a linear form 350 of predictor, $f(\boldsymbol{x})=\boldsymbol{w}^{\top} \boldsymbol{x}$; in addition to the $L_{2}$ norm $\|\boldsymbol{z}\|_{2}=\sqrt{\sum_{j} z_{j}^{2}}$ for the loss function as in OLS, combinations of $L_{1}$, i.e., $\|\boldsymbol{z}\|_{1}=\sum_{j}\left|z_{j}\right|$, and $L_{2}$ are imposed on $\boldsymbol{\omega}$ for regularization to induce sparsity and avoid overfitting. Nonparametric models like K-nearest neighbors (KNN) and Gaussian Process (GP) predict by either $\mathrm{K}$ nearest points or smoothing among available data based on affinity. Multilayer perceptron (MLP) is a popular choice of ANN for power, tariff, and solar predictions [54, 53, which consists of a hidden layer with nonlinear activation functions. It can capture fairly complex scenarios given sufficient data to learn. We refer the readers to [59] for a comprehensive introduction to CI methods, and the accompanying material of this paper for implementation examples.

Forecast combinations. The advantage of combining, according to Hibon and Evgeniou [60, is not "that the best possible combinations perform better than the best possible individual forecasts" (i.e., ex-post), but that "it is less risky in practice to combine forecasts than to select an individual forecasting method" 
(i.e., ex-ante). Given $M$ forecasts from a committee of predictors, $\left\{\hat{y}_{m t}\right\}_{m=1}^{M}$, the simple scheme averages them with equal weights $\frac{1}{M}$. The OLS combination is based on

$$
y_{t}^{\mathrm{OLS}}=c_{\mathrm{OLS}}+\sum_{m=1}^{M} w_{m} \hat{y}_{m t}
$$

where the constant $c_{\mathrm{OLS}}$ and weights $\left\{w_{m}\right\}_{m=1}^{M}$ are learned from past performances of the forecasts. Other schemes, such as the robust regression, which is less sensitive to outliers, the constrained least squares (CLS[c]), which restricts the weights sum up to 1 , and the variance-based combination, which weights the forecasts according to their accuracy measured by the mean squared error (MSE) [60, are also examined in the study.

Above all, in an online adaptive mode, the methods can be reestimated at every time step using a rolling or expanding window, or with more sophisticated adaptive approaches [61, 62].

\subsection{Dispatch Strategies}

The dispatch plan, as has been previously outlined, is developed by solving the mixed-integer program ( $(\underline{\mathrm{P} 0})$ using the forecasts of uncertain variables, subject to power and resilience constraints. Two dispatch strategies, illustrated in Fig. 4. have been implemented in MOD-DR, which are evaluated in Sections 6 and 7 .

Day-ahead dispatch. Like with electricity market bidding, upon receiving predictions of spot prices and solar irradiation, as well as buildings' projected demands and DR signals from the grid on Day 0, MG-C performs $\underline{\underline{\mathrm{P} 0}}$ to prepares a day-ahead dispatch plan and sends it to the generation facility and buildings for review. Iteration of revisions is conceivable until concurrence among the results. The original proposal is amended for actual execution on Day 1. The repair strategy exploits the cheapest sources / destinations of energy immediately available, e.g., storage (if any) or grid, to maintain the power balance. The extent of repair, clearly, is contingent on the intermittency of renewables and forecast errors. 


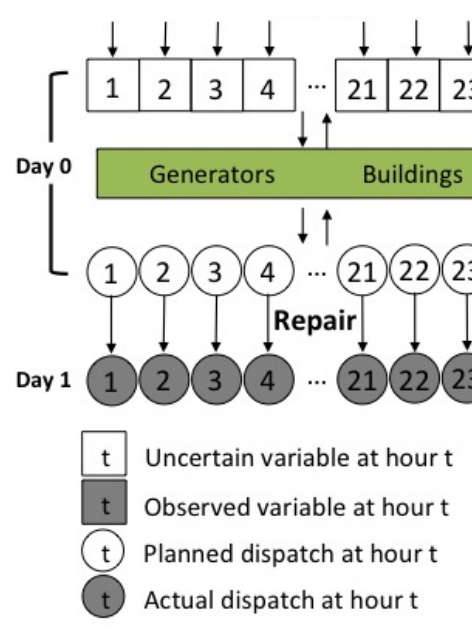

(A) Day-ahead dispatch

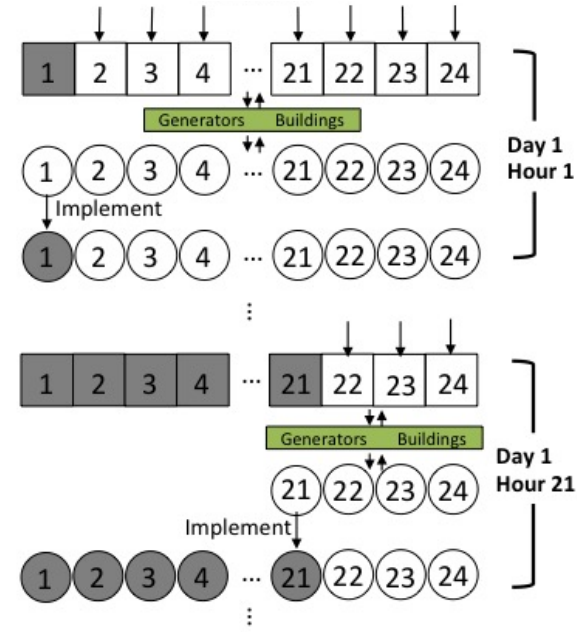

(B) Adaptive dispatch

Figure 4: Illustration of the (A) day-ahead, and (B) adaptive dispatch strategies. For the day-ahead scheme, the plan is devised on Day 0 , and implemented on Day 1 with proper recourse. The adaptive method dynamically plans for the following hours, and updates the proposal as more observations are available.

Adaptive dispatch. The access to real-time information enables the adaptive strategy, which resorts to the receding-horizon, future-discounted variant of $\underline{\underline{\underline{\mathrm{P}}}})$ :

$$
\begin{array}{ll}
\min _{\left\{\boldsymbol{x}_{t}\right\}_{t=1}^{\hat{T}}} & \sum_{t=1}^{\hat{T}} \gamma^{t-1} g_{t}\left(\boldsymbol{x}_{t}, \boldsymbol{z}_{t}, \boldsymbol{\xi}_{t}\right) \\
\text { s.t. } & \boldsymbol{x}_{t} \in \mathcal{X}_{t}\left(\boldsymbol{z}_{t}, \boldsymbol{\xi}_{t}\right), \boldsymbol{z}_{t} \in \mathcal{Z}_{t}\left(\boldsymbol{z}_{t-1}, \boldsymbol{x}_{t}\right), \forall t=1, \ldots, \hat{T}
\end{array}
$$

where $g_{t}\left(\boldsymbol{x}_{t}, \boldsymbol{z}_{t}, \boldsymbol{\xi}_{t}\right)=f_{t}^{\mathrm{Ope}}\left(\boldsymbol{x}_{t}, \boldsymbol{\xi}_{t}\right)+f_{t}^{\mathrm{Mtn}}\left(\boldsymbol{x}_{t}, \boldsymbol{z}_{t}, \boldsymbol{\xi}_{t}\right)-R_{t}^{\mathrm{DR}}-\lambda_{\text {util }} u_{t}\left(\boldsymbol{x}_{t}\right)$ is the original objective term, further discounted by $\gamma \in[0,1]$, and $\hat{T} \leq T$ denotes the receding horizon. To be specific, at the start of Hour 1 of Day 1, $\underline{\underline{\mathrm{P} 1}}$ is carried out with $\hat{T}=23$, using the current observation $\boldsymbol{\xi}_{1}$ and the forecasts of $\left\{\boldsymbol{\xi}_{t}\right\}_{t=2}^{24}$ for the rest of the day. Then, assuming no abrupt changes during Hour 1, the plan is executed. The procedure is reiterated for the subsequent hours, while receding the planning horizon $\hat{T}$ and only operating the part of the strategy for the current point, as clarified in Fig. 4, till the end of the 
working day. As uncertainty over renewables is resolved by observation for the current hour, the need for repair is obviated. The mechanism of $\gamma$ resembles that of the dynamic programming 63 : for $\underline{\underline{\mathrm{P}} 0,}, \gamma=1$, and the planner becomes increasingly nearsighted as $\gamma$ shrinks. 4

While the adaptive strategy maintains more frequent communication with the generation facility and DR loads compared with the day-ahead scheme, it delivers the benefits of improved accuracy of forecasts, cost effectiveness of dispatch, and responsiveness to the grid. Depending on additional constraints like the computational capability in solving large-scale MILP and inter-temporal parameters of generators, e.g., minimum run time and notification time, both are viable for practical deployment [20, 33, 37, 29, 64.

\section{Experimental Setup}

The study focuses on the district level heating and cooling, where the thermal and electric energy is supplied by the microgrid for a cluster of buildings. We give an overview of the publicly available datasets and our implementation of MOD-DR .

\subsection{Dataset}

Building loads. In practice, buildings with compatible and sympathetic load profiles serve to level the aggregated energy demand, thus improving both the operating efficiency and the overall MG economics. The load data are retrieved from the Open Energy Information (OpenEI), for a research facility (Bld:1) 55 a large hotel (Bld:2), and a commercial building (Bld:3) 6 , though more buildings can be readily incorporated. For the electricity loads, as is shown in Fig. 5 , the load types are inferred primarily from descriptions, e.g., demands for the data

\footnotetext{
${ }^{4}$ At the limit of $\gamma=0$, we keep to the convention that $0^{0}=1$, in which case the planner only cares about the cost for the current hour, disregarding any future risks or arbitrage events.

5 NREL RSF Measured Data 2011 accessed: 10/2016

6 OpenEl Load Protiles accessed: 10/2016
} 

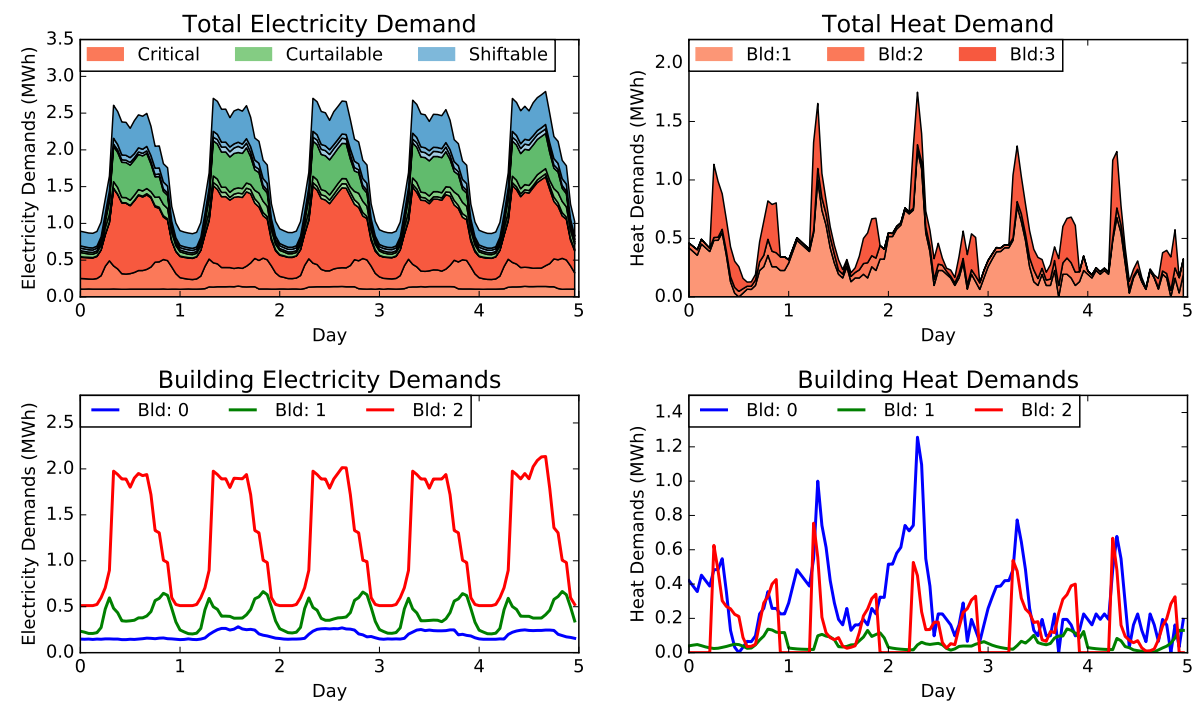

Figure 5: Left panel: Electricity load profiles (top), which display the critical loads (red), curtailable loads (green), and shiftable loads (blue), for three buildings (different shadings; total electricity demands for each building (bottom). Right panel: Heat demands for the buildings under study, plotted with stacked (top) and separate lines (bottom).
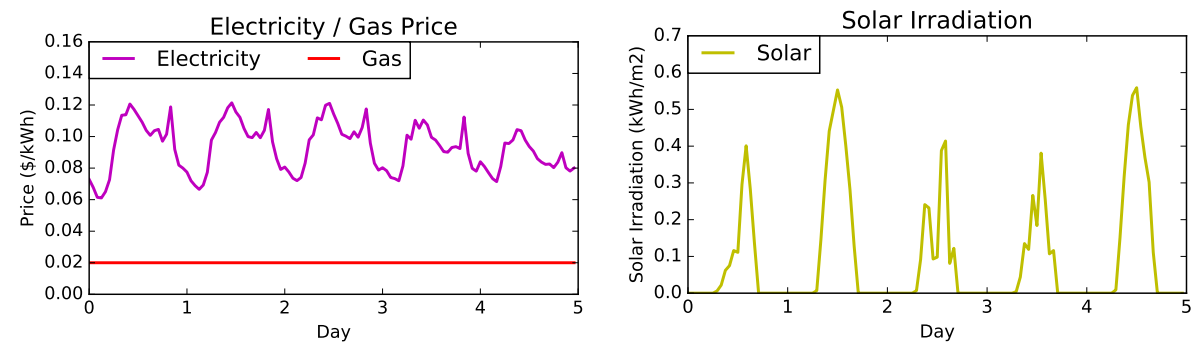

Figure 6: Electricity and natural gas tariffs Figure 7: Solar irradiation measured by the for industrial customers on several days, where GHI index $\left(\mathrm{kWh} / \mathrm{m}^{2}\right)$ on several days of the the spark spread is mainly driven by the daily study period, which clearly exhibits diurnal fluctuation of electricity prices. patterns.

center are critical, ventilation and lighting are curtailable, and plug-loads are shiftable. During the period of study, i.e., January and February, the thermal loads are predominantly for heating (Fig. 5). Throughout the paper, it is 
assumed that the loads are given, though in practice a forecasting module can be employed based on Section 4.1 (especially the forecast combination method which is often used for load prediction [54]).

Electricity and gas prices. The electricity spot price is accessed from the National Grid Online Databas $\AA^{7}$ for the period of Jan. 1 to Jul. 24, 2016 in New York, adapted to be similar to the California wholesale market, whose daily fluctuation is exhibited in Fig. 6. The natural gas price, obtained from the U.S. Energy Information Administration ${ }^{8}$ is assumed to be at a constant level of $0.02 \$ / \mathrm{kWh}$ throughout the month. The difference between the electricity and gas price, a.k.a., the "spark spread", has an underlying daily cycle driven by variations in the average electricity price, which can be potentially exploited through the incorporation of heat storage.

Solar irradiation. The TMY3 dataset is derived from the National Solar Radiation Data Base (NSRDB) archives for 1020 locations from 1961 - 1990 and 1991 - 2005 [65]. We query the data for Oakland, California in the year 2005, and use the Global Horizontal Irradiance (GHI) index 9 to determine the PV outputs [53, as is plotted in Fig. 7 for several days.

\subsection{Implementation}

The implementation of MOD-DR is based on our previous work [19] on MG long-term capital investment, which modeled generator and storage technologies. The platform is realized in Python, an object-oriented programming language with cross-platform compatibility and extensibility to hardware actuators and databases like MySQL and Cassandra for real-time controllability. The core MILP programs $(\underline{\underline{\mathrm{P}}})$ and $(\underline{\mathrm{P} 1})$ for dispatch are built and solved by Gurobi

\footnotetext{
7 National Grid Online Database, accessed: 10/2016 $\varepsilon$ U.S. Energy Information Administration accessed: 10/2016

${ }^{\mathrm{S}} \mathrm{GHI}$, measured in $1 \mathrm{kWh} / \mathrm{m}^{2}$, is the total amount of direct and diffuse solar radiation received on a horizontal surface during the 60 -minute period.
} 

i7 CPU and 16 GB RAM memory.

\section{Scenario Analysis}

The objective of this section is to analyze the effects of uncertainty on dispatch and the DR potential for load shaping. Four scenarios are designed, which focus on (1) the spot price and (2) renewable energy prediction, (3) the integration of energy storage, and (4) the operation of the DR program.

Throughout the discussions, the concept of additional operational costs is applied to refer to the added costs compared to the minimum among the set of candidates, which often corresponds to the oracle with perfect predictions and optimal combination of DG and storage. The specifications of technologies, listed in Table 1 (c.f., 99), are employed in both the current section and the subsequent case study. For the following four studies, the day-ahead dispatch strategy $\mathrm{P} 0$ is adopted for analysis. The adaptive strategy is employed in Section 7 for comparison.

\subsection{The Effect of Uncertainty on Dispatch Optimality}

The pool price exposure and renewable volatility give rise to the increased variability in real-time resource commitment and dispatch. The object of the controlled experiment is to assess the forecasting approach of MOD-DR, as well as the practical implications of storage in the presence of uncertainty.

465

Study 1: The effect of electricity price uncertainty

To begin with, we study the multiple predictors in Section 4.1 which are trained and tested on two subsequent months of data. The results for day-ahead prediction (Fig. 8 and Table 2) are reported in root mean squared error (RMSE),

470 given by $\sqrt{\frac{1}{24} \sum_{t=1}^{24}\left(f_{t}\left(\boldsymbol{x}_{t}\right)-y_{t}\right)^{2}}$, which compares the estimation $f_{t}\left(\boldsymbol{x}_{t}\right)$ with the true price $y_{t}$ throughout the day [54]. For CI and forecast combinations, separate estimators for each hour of the day have been learned, using $\boldsymbol{x}_{t}$ as 
Table 1: Specification of parameters for distributed generators and storage used in the experiments, which also includes thermal efficiency of pipelines in load follow mode, where the indices are circled in Fig. 3 (A).

\begin{tabular}{|c|c|c|}
\hline Technology & \multicolumn{2}{|c|}{ parameter: value } \\
\hline PV & solar efficiency $\eta^{\mathrm{PV}}: 0.15$ & unit out ratio $e_{\mathrm{rat}}^{\mathrm{PV}}: 0.15$ \\
\hline Solar thermal & solar efficiency $\eta^{\text {SolThem }}: 0.69$ & unit out ratio $e_{\text {rat }}^{\text {SolThem }}: 0.1$ \\
\hline \multirow[t]{2}{*}{ CHP } & $E_{\text {rat }, k}^{\mathrm{CHP}}(\mathrm{MW}): 1.5,2,3,4$ & partial loads $L_{k}^{\mathrm{CHP}}: 0.1$ \\
\hline & $\mathrm{HER}_{k}: 1.2,1.45,1.5,1.5$ & $\eta_{k}^{\mathrm{CHP}}: 0.4,0.45,0.45,0.5$ \\
\hline \multirow[t]{3}{*}{ Electric battery } & charging efficiency $\eta_{\text {chg }}^{\text {Bat }}: 0.9$ & discharging eff. $\eta_{\text {dis }}^{\text {Bat }}: 0.9$ \\
\hline & standing loss $\eta_{\text {decay }}^{\text {Bat }}: 0.001$ & max charge rate $\tau_{\text {chg }}^{\text {Bat }}: 0.25$ \\
\hline & max discharge rate $\tau_{\text {dis }}^{\text {Bat }}: 0.25$ & $\min \mathrm{SOC} \mathrm{SOC}_{\mathrm{min}}^{\mathrm{Bat}}: 0.1$ \\
\hline \multirow[t]{3}{*}{ Heat tank } & charging efficiency $\eta_{\text {chg }}^{\mathrm{HT}}: 0.9$ & discharging eff. $\eta_{\text {dis }}^{\mathrm{HT}}: 0.9$ \\
\hline & standing loss $\eta_{\text {decay }}^{\mathrm{HT}}: 0.01$ & max charge rate $\tau_{\text {chg }}^{\mathrm{HT}}: 0.25$ \\
\hline & $\max$ discharge rate $\tau_{\mathrm{dis}}^{\mathrm{HT}}: 0.25$ & $\min \mathrm{SOC} \mathrm{SOC} \mathrm{Hin}_{\mathrm{min}}^{\mathrm{HT}}: 0$ \\
\hline \multirow[t]{3}{*}{ Cool tank } & charging efficiency $\eta_{\text {chg }}^{\mathrm{CT}}: 0.9$ & discharging eff. $\eta_{\text {dis }}^{\mathrm{CT}}: 0.9$ \\
\hline & standing loss $\eta_{\text {decay }}^{\mathrm{CT}}: 0.01$ & max charge rate $\tau_{\text {chg }}^{\mathrm{CT}}: 0.25$ \\
\hline & max discharge rate $\tau_{\text {dis }}^{\mathrm{CT}}: 0.25$ & $\min \mathrm{SOC} \mathrm{SOC}_{\min }^{\mathrm{CT}}: 0$ \\
\hline Electric boiler & \multicolumn{2}{|c|}{ coefficient-of-performance COP ${ }^{\text {ELEBoil }}: 5.0$} \\
\hline NG boiler & \multicolumn{2}{|l|}{ thermal efficiency $\eta^{\text {NGBoil }}: 0.82$} \\
\hline Electric chiller & \multicolumn{2}{|c|}{ coefficient-of-performance COP ${ }^{\text {ELEChill }}: 5.47$} \\
\hline NG chiller & \multicolumn{2}{|l|}{ thermal efficiency $\eta^{\text {ELEChill }}: 0.65$} \\
\hline Absorption chiller & \multicolumn{2}{|c|}{ coefficient-of-performance COP ${ }^{\mathrm{ABSChill}}: 1.0$} \\
\hline \multirow[t]{2}{*}{ Pipelines } & \multicolumn{2}{|c|}{ heating efficiency $\left\{\eta_{j}^{\text {ld,heat }}\right\}_{j=1}^{5}: .99, .98, .988, .977, .977$} \\
\hline & \multicolumn{2}{|c|}{ cooling efficiency $\left\{\eta_{j}^{\text {ld,heat }}\right\}_{j=1}^{5}: .985, .986, .987, .984, .979$} \\
\hline
\end{tabular}


the prices for the previous two days. As can be seen, forecast combinations, i.e., OLS [c], CLS [c], and Robust [c] are significantly better than the baseline methods, and also consistently outperform the individual models.

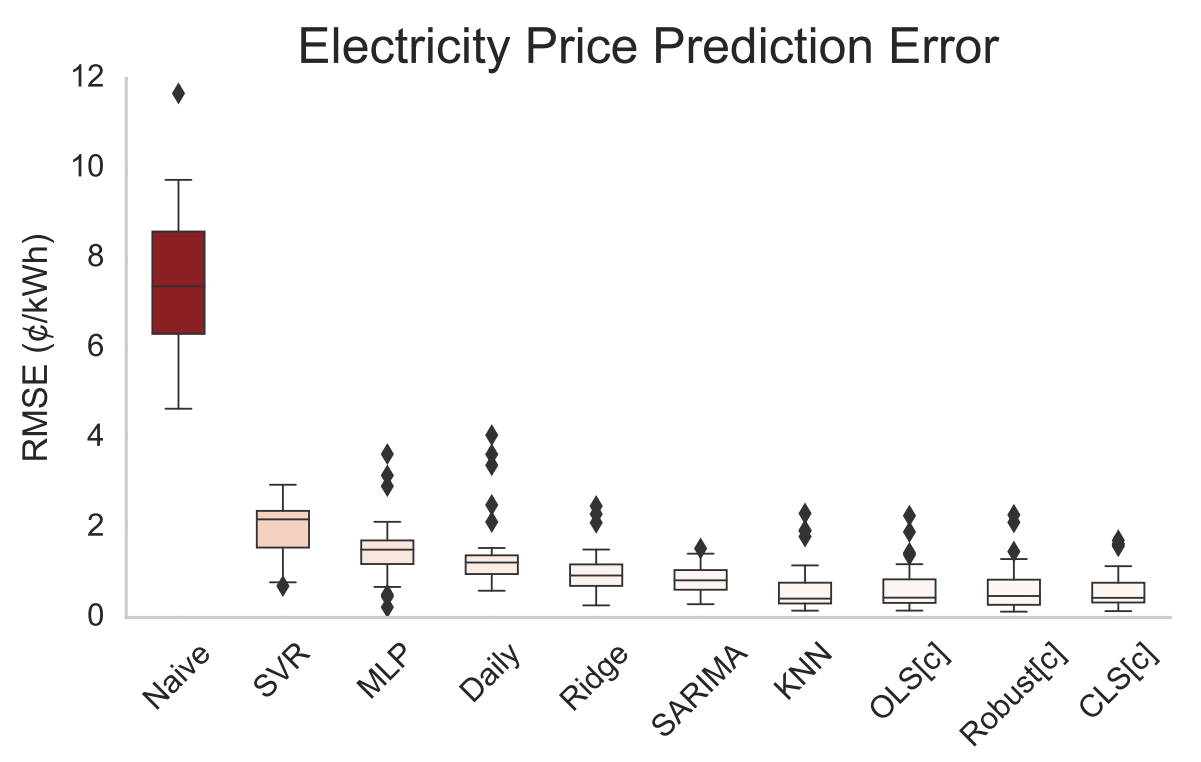

Figure 8: Boxplots of the spot price forecast RMSE $(\$ / \mathrm{kWh})$ for multiple methods. The box shows the quartiles, and the whiskers extend to show the rest of the distribution, while the black dots indicate outliers.

The integration of each forecaster with MOD-DR is examined under the prototype, MG1, which consists of a NG boiler (1MW), an electric boiler (1MW), an assembly of CHPs (0.5, 1, 1.5, 2 MW capacities), a heat tank (2MWh) and a electric battery $(4 \mathrm{MWh})$, whose parameters are listed in Table 1 . The operational surcharge is derived for each candidate by collating with the oracle, which is able to dispatch based on the true spot price (Fig. 10 and Table 2). The daily dispatch plan is shown in Fig. 9, which demonstrates that even though there is a mismatch between prediction and true spot prices, CLS [c] can capture the two peaks, and thus schedule the electric storage to exploit the time-differentiated 485 prices.

Further, the effect of forecasting error on operational cost is demonstrated 
Table 2: Cost analysis statistics for Study 1 and 2, including the RMSE of the forecast, and the corresponding additional operation cost (one standard deviation is enclosed in parentheses). Models incorporated in forecast combinations include KNN, Ridge, SVR, MLP, and Naïve methods. The monthly costs for the Oracle with perfect prediction are $\$ 36.8 \mathrm{~K}$ and $\$ 25.2 \mathrm{~K}$ in Study 1 and 2, respectively. In Study 2, the monthly cost for the case without PV is $\$ 37.7 \mathrm{~K}$.

\begin{tabular}{|c|c|c|c|c|}
\hline \multirow[b]{2}{*}{ Forecaster } & \multicolumn{2}{|c|}{ S1: Electricity price } & \multicolumn{2}{|c|}{ S2: Solar GHI } \\
\hline & $\begin{array}{c}\text { RMSE } \\
(\mathrm{c} / \mathrm{kWh})\end{array}$ & $\begin{array}{l}\text { Add. cost } \\
(\mathrm{k} \$ / \text { month })\end{array}$ & $\begin{array}{c}\text { RMSE } \\
\left(\mathrm{kWh} / \mathrm{m}^{2}\right)\end{array}$ & $\begin{array}{l}\text { Add. cost } \\
(\mathrm{k} \$ / \text { month })\end{array}$ \\
\hline Naïve & $7.9(2.3)$ & $.5(.5)$ & $.31(.09)$ & $2.5(2.8)$ \\
\hline Ridge & $1.0(.5)$ & $.7(.6)$ & $.09(.04)$ & $3.1(3.1)$ \\
\hline KNN & $.7(.5)$ & $.5(.6)$ & .09 (.03) & $3.0(2.6)$ \\
\hline MLP & $1.5(.7)$ & $1.3(.6)$ & $.09(.03)$ & $3.0(3.0)$ \\
\hline SVR & $2.0(.6)$ & $1.4(.7)$ & $.09(.03)$ & $3.0(3.0)$ \\
\hline OLS $[\mathbf{c}]$ & $.7(.5)$ & $.5(.5)$ & $.09(.02)$ & $2.5(2.2)$ \\
\hline CLS $[c]$ & $.6(.4)$ & $.4(.4)$ & $.09(.03)$ & $3.0(2.8)$ \\
\hline Robust $[\mathbf{c}]$ & $.6(.5)$ & $.5(.6)$ & .09 (.03) & $2.4(2.3)$ \\
\hline
\end{tabular}
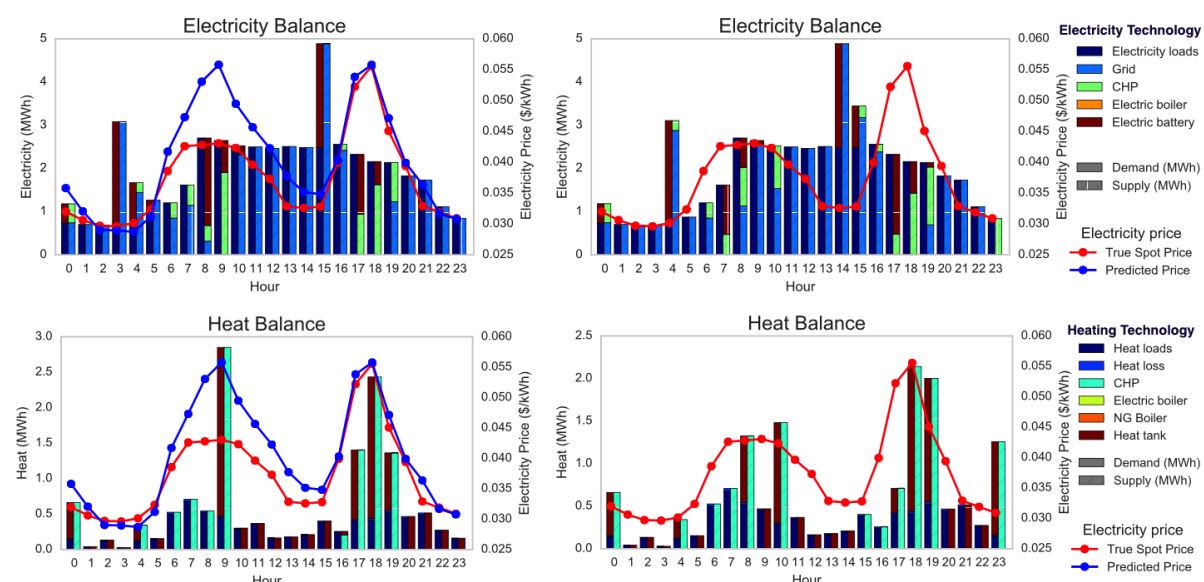

Hour

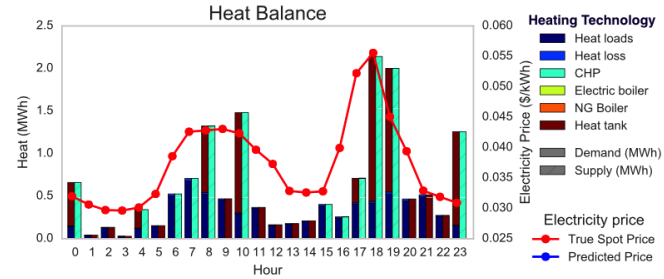

Figure 9: Electricity and heat balances for day-ahead dispatch based on CLS forecasts (left) and true (right) electricity price for S1 under Study 1. The effect of forecast errors can be seen, for instance, at hour 9, when CHP is turned on in the face of predicted higher spot price.

in Fig. 11. The positive correlation is consolidated by the Pearson coefficient of .82. The p-value of the test is less than $1 \mathrm{e}-5$, which indicates that the probability of observing a phenomenon as extreme as the current case under the non- 


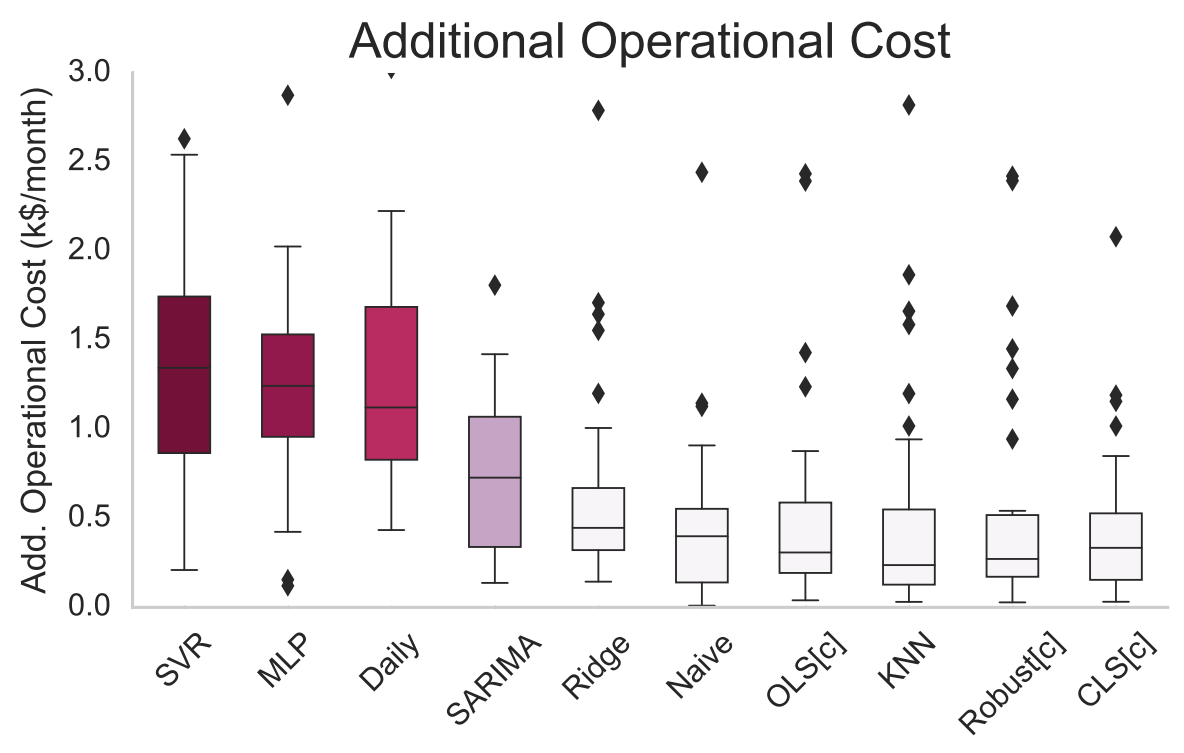

Figure 10: Boxplots of the additional operational cost of MG1 when different electricity price forecasters are integrated.

correlation condition is exceedingly small; in other words, the null-hypothesis of non-correlation is rejected with statistical significance 10

Our conclusion is that the fluctuation of electricity spot price exposes $M G$ to operational suboptimality and financial risk, inasmuch as the variable can be predicted with accuracy. This result is in alignment with the findings from [22, 32]; however, their studies incorporated the situation with only electricity loads and no distributed generation capacity, and the forecasting errors were simulated from a noise model. Our result, in comparison, applies to MG with both electric and thermal loads, and the electricity price is forecasted based on historical data with current predictors.

${ }^{10}$ The Pearson coefficient between two variables $y_{1}(t)$ and $y_{2}(t)$ is given by:

$$
\frac{\sum_{t=1}^{T}\left(y_{1}(t)-\bar{y}_{1}\right)\left(y_{2}(t)-\bar{y}_{2}\right)}{\sqrt{\sum_{t=1}^{T}\left(y_{1}(t)-\bar{y}_{1}\right)^{2}\left(y_{2}(t)-\bar{y}_{2}\right)^{2}}}
$$

where $\bar{y}_{1}$ and $\bar{y}_{2}$ are the sample mean of $y_{1}$ and $y_{2}$, respectively. 


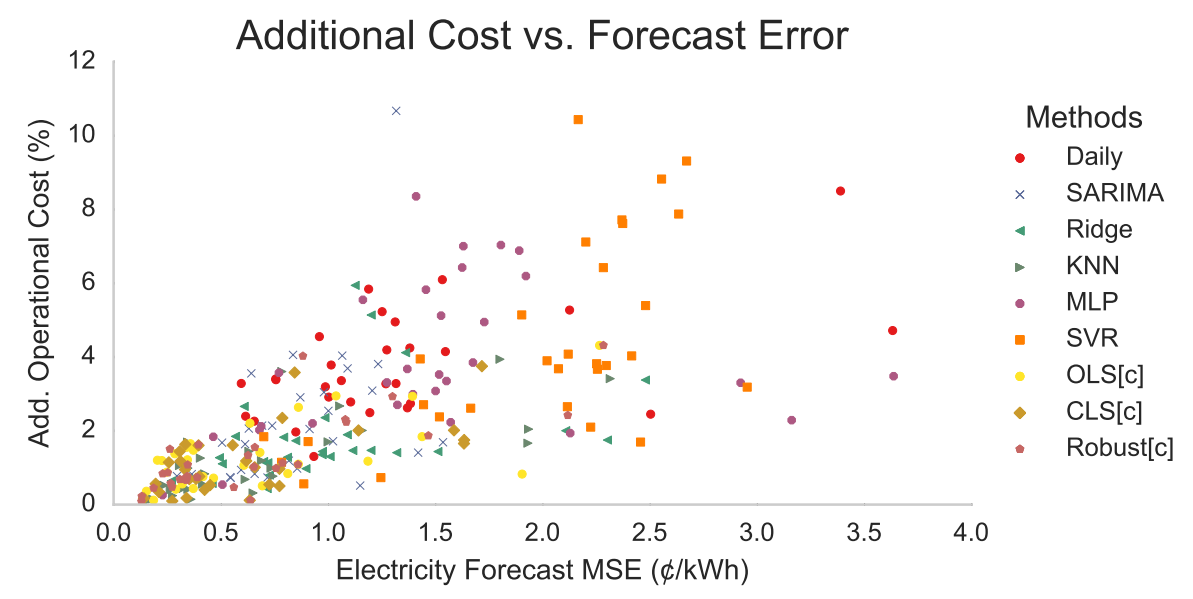

Figure 11: Scatter plots of the electricity forecast error against the operational surcharge for the list of predictors under study.

Study 2: The effect of renewable uncertainty

It can be observed in Fig. 7 that solar irradiation exhibits strong diurnal patterns. As in Study 1, day-ahead predictors are assessed (Table 2), among which forecast combinations consistently outperform other candidates. Additionally, prediction reliability, as is reflected in the lowered standard deviation, is enhanced by encompassing all possible traces.

The evaluation of forecasting methods is conducted for system MG2, which additionally equip MG1 with PV $\left(0.05 \mathrm{~km}^{2}\right.$ installation area) and solar thermal $\left(0.01 \mathrm{~km}^{2}\right)$. We assume the access to the next-day electricity price and do not engage in load shaping to limit the coupling effect. Clearly, a repair strategy, as detailed in Section 4.2, is in place to maintain power balance. The integration of renewables during the planning stage with proper forecasts can drive down the cost by $\$ 8 \mathrm{~K}$ per month under the current solar profile (Table 2), which can also be verified from the daily dispatch plans in Fig. 12 that make full use of solar energy to meet the MG loads.

Forecasting accuracy, furthermore, directly contributes to the efficiency of 

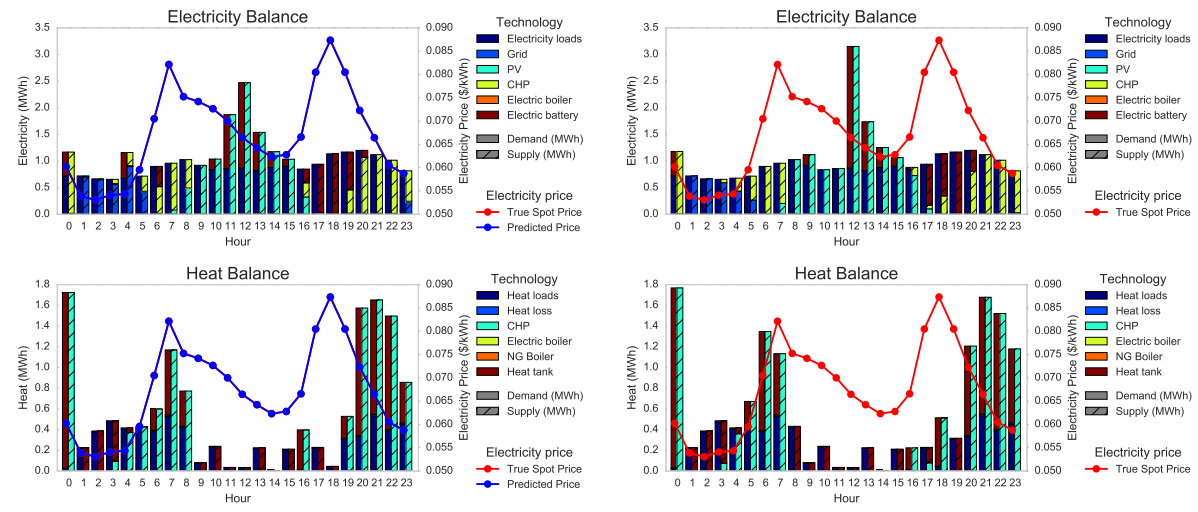

Figure 12: Electricity and heat balances for day-ahead dispatch based on CLS forecasts (left) and true (right) solar energy, when the electricity price is assumed to be known a priori. The effect of forecast errors can be inspected at hour 11 (over-forecasting) and 12 (underforecasting). In the former case, more electricity needs to be purchased from the grid, while in the latter case the renewable is under-utilized.

production planning (Fig. 13), as verified by the Pearson test with a correlation coefficient of 0.17 and p-value 5e-3 indicating statistical significance. If ensuring that the solution can survive a certain degree of variability is of concern, a common strategy adopted in unit commitment 20] is to systematically underestimate the solar power, which can be readily accomplished in forecast combinations by considering the no-PV situation.

In summary, the intermittent renewables can be effectively harnessed by forecasting to improve the economy. Morais et al. [10] demonstrates the dispatch with forecasted solar profile of a MG in Budapest Tech, which includes, among others, PV panels (peak power 150W) and an electric battery (max charging/discharging power $200 \mathrm{~W} / 50 \mathrm{~W}$ ). While they only examined the effect of forecasting error on operations, such as the battery charging of excess power due to under-prediction, our results (Fig. 13 , Table 2) report statistical evidence of the economic impacts.

Study 3: The integration of energy storage

With the growing penetration of renewable energy resources and a shift 


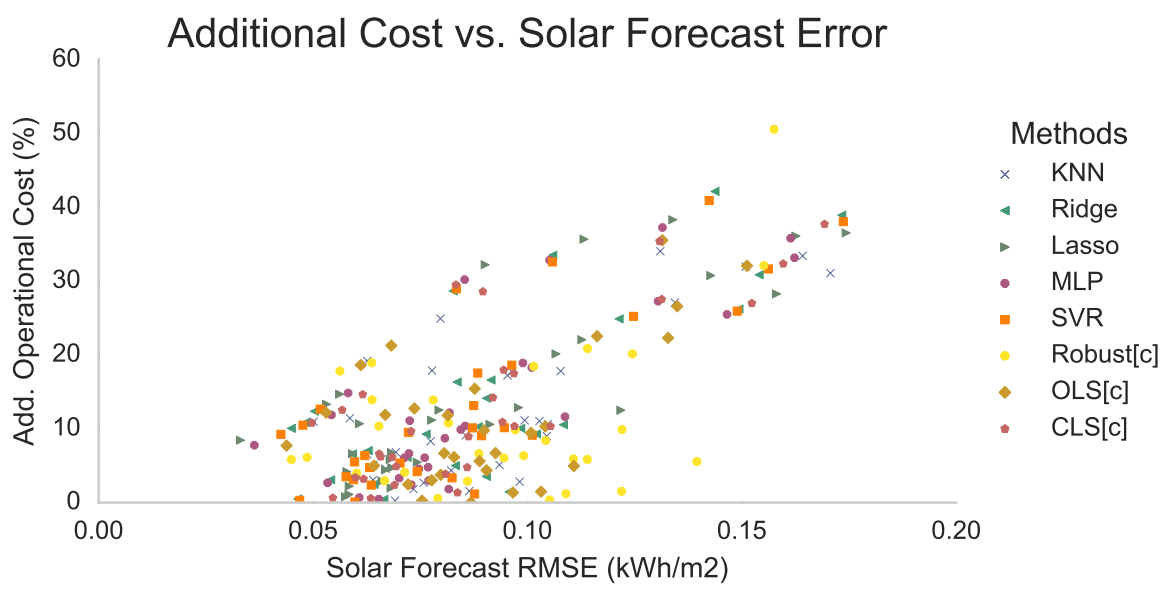

Figure 13: Scatter plots of the additional operational costs (compared with the Oracle) vs. the forecast errors for different methods.

to a much more dynamic electric infrastructure, the benefits that storage can deliver, as suggested in [66], range from electricity supply and ancillary services, to transmission support and renewables capacity firming. It is evident thus far (Fig. 9 and 12 that the ability of storage to make cost reductions depends in large part on:

- Time-of-use (TOU) management: storage is charged during off-peak hours and discharged during times when on-peak TOU energy prices apply.

- Renewables time-shift: storage operates in conjunction with renewable generation to enable the use of low-value energy generated during off-peak times to offset other purchases when it is more valuable.

Importantly, the object is to study the amount of benefits that storage can 545 reap in the face of uncertainty. The system MG2 from the previous test is supplied with different sizes of electric battery and / or heat tank, which are dispatched for a month when both the electricity price and the solar irradiation are forecasted during day-ahead planning. According to the result, the lowest operational cost is achieved when both the heat and electricity storage are present 

compared with other situations when only the heat tank (Case B) or the electric battery (Case C) is present for the Naïve and CLS forecasters to evaluate the costs (Fig. 14 and Table 3).

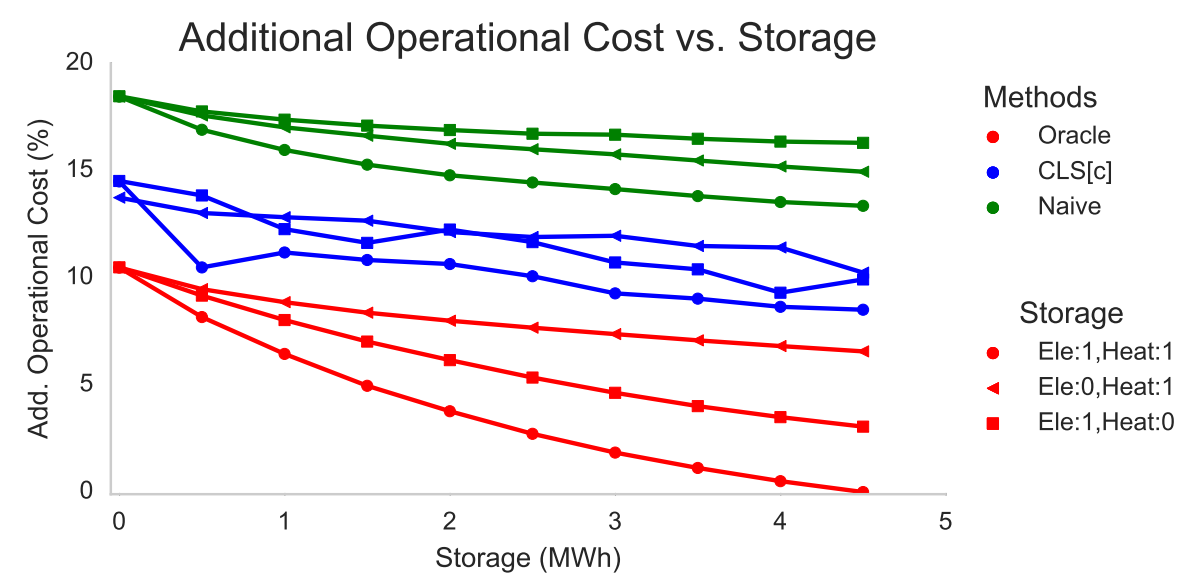

Figure 14: Additional operational cost for day-ahead dispatch based on oracle, CLS[c] and Naïve forecasters of solar and electricity prices. Each point represents 30-day average, where the colors indicate dispatch strategies and shapes correspond to different cases (A, B, C).

As it can be seen, increasing the capacity of storage can bring greater savings in all cases $(\mathrm{A}, \mathrm{B}, \mathrm{C})$, though with marginal diminishing returns. Interestingly, while the storage for electricity (case C) seems more favorable than heat (case B) under perfect prediction, likely because the potential of TOU management for the electricity is higher, the effect is not as pronounced when both the electricity price and solar are forecasted, especially for the Naïve method, which tends to perform poorly (Table 2). Indeed, with better predictions like CLS, further reductions can be achieved.

Prior work [45, 15, 19] tackled the capacity planning of storage based on long-term valuation, where uncertainty in variables are not considered. Out results indicate that the fulfillment of the potentials is contingent on uncertainty inherent in practical dispatch. Díaz and Moreno 13 investigated the valuation 
Table 3: Operational charge for several schemes as the storage capacity varies, when both heat and electricity storage (case A), or only one (B for heat and C for electricity) is present. The cost is compared with the Oracle with $4.5 \mathrm{MWh}$ heat and electricity storage, i.e., case A, whose daily cost is averaged at $\$ 791.3$. For instance, the average daily cost for CLS[c] when both electricity and heat storage have capacities of $2 \mathrm{MWh}$ is $\$ 791.3+\$ 84=\$ 875.3$. Both the 30-day average and standard deviation (in parenthesis) are shown.

\begin{tabular}{|c|c|c|c|c|c|c|}
\hline \multirow{2}{*}{} & \multicolumn{5}{|c|}{ Capacity (MWh) } \\
\cline { 2 - 7 } & 0 & 1 & 2 & 3 & 4 \\
\hline \multirow{3}{*}{ A } & Naïve & $146(18.4)$ & $126(16.0)$ & $117(14.8)$ & $112(14.1)$ & $107(13.5)$ \\
\cline { 2 - 7 } & CLS[c] & $115(14.5)$ & $88(11.2)$ & $84(10.6)$ & $73(9.3)$ & $68(8.6)$ \\
\cline { 2 - 7 } & Oracle & $83(10.5)$ & $51(6.4)$ & $30(3.8)$ & $15(1.8)$ & $4(0.5)$ \\
\hline \multirow{3}{*}{ B } & Naïve & $146(18.4)$ & $135(17.0)$ & $129(16.2)$ & $125(15.8)$ & $120(15.2)$ \\
\cline { 2 - 7 } & CLS[c] & $109(13.7)$ & $101(12.8)$ & $96(12.1)$ & $95(12.0)$ & $90(11.4)$ \\
\cline { 2 - 7 } & Oracle & $83(10.5)$ & $70(8.9)$ & $63(8.0)$ & $58(7.4)$ & $54(6.8)$ \\
\hline \multirow{3}{*}{ C } & Naïve & $146(18.5)$ & $137(17.4)$ & $134(16.9)$ & $132(16.7)$ & $129(16.4)$ \\
\cline { 2 - 7 } & CLS[c] & $115(14.5)$ & $97(12.3)$ & $97(12.2)$ & $85(10.7)$ & $74(9.3)$ \\
\cline { 2 - 7 } & Oracle & $83(10.5)$ & $64(8.0)$ & $49(6.2)$ & $37(4.6)$ & $28(3.5)$ \\
\hline
\end{tabular}

of CHP and thermal storage under uncertain energy prices, and reported the link between the economic scales of CHP and thermal storage. Our study, in addition, presented the influence of forecasting model and accuracy on storageaugmented system operation.

\subsection{The Effect of DR for Load Shaping}

The value and necessity of DR as a flexibility means has been widely recognized among stakeholders and policy makers, whose objectives can be divided into two categories, i.e., energy adjustments (e.g., strategic conservation and strategic load growth) and power adjustments (e.g., peak clipping, valley filling, load shifting and flexible load shape) [41, 43, 42, 67, 6]. The latter, in particular, requires the active participation of consumers, who are rather passive nowadays [6]. The object is, therefore, to investigate the engagement of DR facilitated by MOD-DR based on real-time pricing. 
The consumption preferences of individual buildings can be revealed to MG$\mathrm{C}$ through both the utility function and the load mix, as depicted in Fig. 5 . From the control perspective, the shiftable load needs some planning and thus benefits from static signals notified well in advance, while the curtailable load can respond to dynamic signals more swiftly. The utility parameters, for simplicity, are shared among all three buildings for heating, cooling, and electricity loads, with $\theta_{0}, \theta_{1}, \theta_{2}=0,0.5,1$, and $v_{1}, v_{2}=1,0.2$. Both systems under study, MG4.1 and MG4.2, are electricity-only, i.e., no thermal loads, equipped with four types of CHP $(0.5,1,1.5,2 \mathrm{MW})$, and MG4.2, in addition, has an electric battery $(2 \mathrm{MWh})$. As in [41, perfect prediction is assumed, which is the best case scenario for DR, and hence ideal for impact studies.

During the one month of the experiment, DR is called when the wholesale price is above the strike price, i.e., the price at which the customer has agreed to provide demand response [16, which is chosen as the third quartile of history data. On a particular day, upon gathering of information from buildings, generators, and the grid, MG-C performs MOD-DR to come up with a dispatch proposal to be implemented on the next day. A viable solution is exemplified in Fig. 15, which represents $70.6 \%$ peak load reduction from the original $5.8 \mathrm{MWh}$ peak hour demand.

600 Compared with dispatching without the engagement with DR, MOD-DR explores the feasibility of seeking trade-offs between building satisfaction and operational savings. Indeed, the flexibility is realized by consciously tuning the parameter $\lambda_{u t i l}$, as is shown in Fig. 16, which unveils a smooth spectrum of optimal resolutions gauged by the loss of satisfaction, measured by the percentage of utility loss compared to the case without load shaping, and the additional operational cost. It is up to the MG-C committee to decide upon the level of DR commitment, by potentially taking advantage of the fact that substantial cost saving can be achieved without sacrificing customer contentment. Additionally, system MG4.2 with the electric battery stands on the Pareto frontier, though

Taking into account demand elasticity, consumption patterns, as well as 


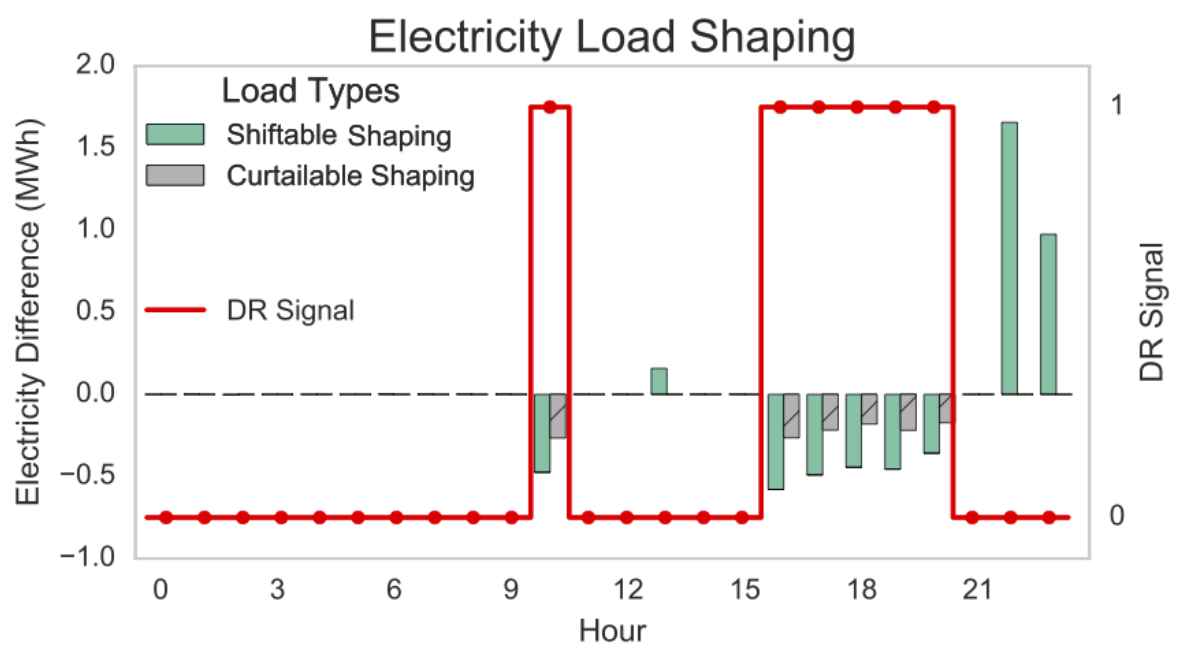

Figure 15: Electricity load shaping after implementing DR, where negative / positive values indicate reduction / addition from the original demands. When there is no DR event, all the curtailable loads are satisfied, and it is possible to consume more than the original profile to compensate for the shifted loads in previous hours.

grid signals and generation profiles, MOD-DR is able to maintain customer's satisfaction and coincidentally produce the desired changes in the district loads through an optimal utility-cost trade-off. Compared to the study by Kim and

Giannakis 44, we consider the demand on the buildings level rather than device level, and include thermal loads in addition to electricity loads satisfied by CHP and other DGs. Further, the proposed utility cost trade-off curve (Fig. 16) is useful to choose between DR strategies.

\section{Microgrid Case Study}

The objective is to undertake an assessment of MOD-DR for the capability of demand response utility trade-off and operational cost savings, with greater renewable penetration and price uncertainty in a practical-oriented setting.

The prototype system, derived from the University of California, San Diego (UCSD) microgrid for the capacities of generators and renewables [68], as well 


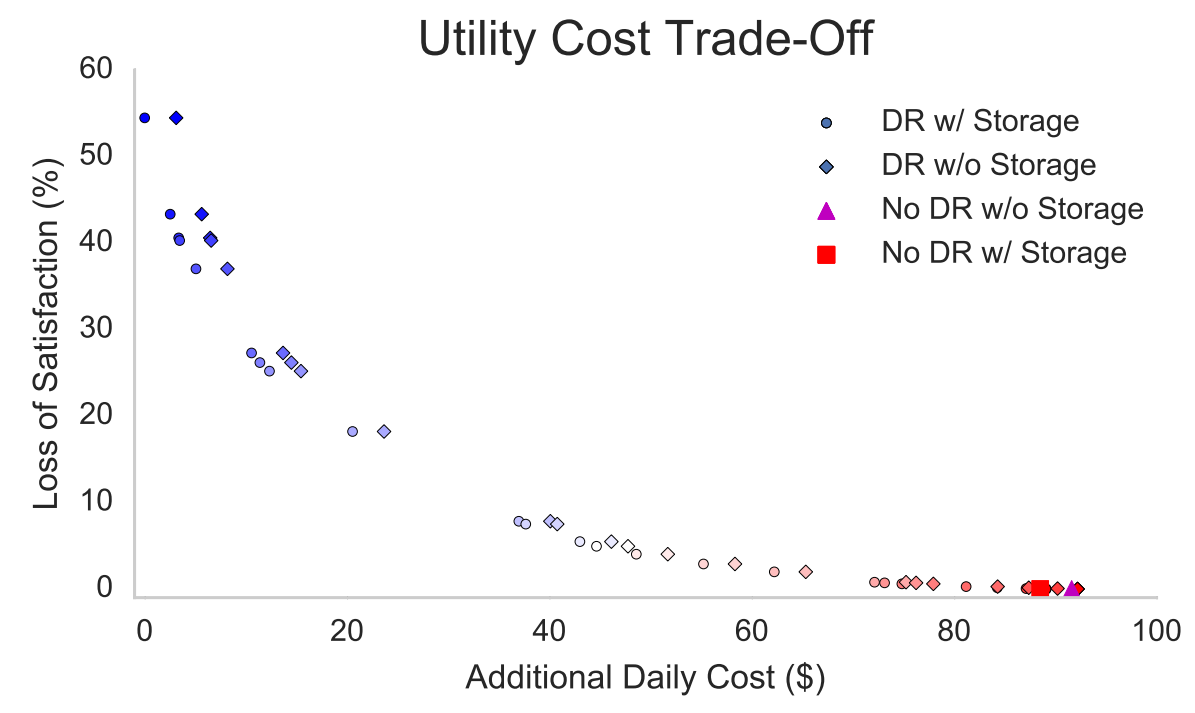

Figure 16: Loss of satisfaction and additional cost trade-off for a typical day, shown for systems with and without storage or DR under perfect predictions. The utility parameters, whose values are color coded (the redder the larger), ranges from 1 to 400, equally spaced in log scales. Baseline for maximum satisfaction is MG4.2 without DR; baseline for minimum cost is MG4.2 with DR and $\lambda_{u t i l}=0$.

as the British Columbia Institute of Technology (BCIT) campus for the DRenabled load control 69, is comprised of CHP plants $(1.5,2,3,4 \mathrm{MW})$ that work in conjunction with an absorption chiller (10MW), a NG boiler (5MW) and chiller (10MW), an electric boiler (10MW) and chiller (10MW), storage for electricity (4MWh), heat (4MWh), and cooling (4MWh), in addition to a PV site $\left(.05 \mathrm{~km}^{2}\right)$, for the provision of electric and thermal energy. While the solar profile is maintained, other data from the previous section are adapted to comply with the requirements, e.g., electricity and cooling loads are scaled up by a factor of 5 and 10 (Fig. 18). The evaluation spans the month of April, when both heating and cooling demands are present.

Uncertainty in both electricity price and solar irradiation are assumed. The dispatch strategies under analysis include day-ahead Oracle, i.e., perfect predictions, with and without load shaping DR, day-ahead $\underline{\underline{P 0}}$ and adaptive schemes 


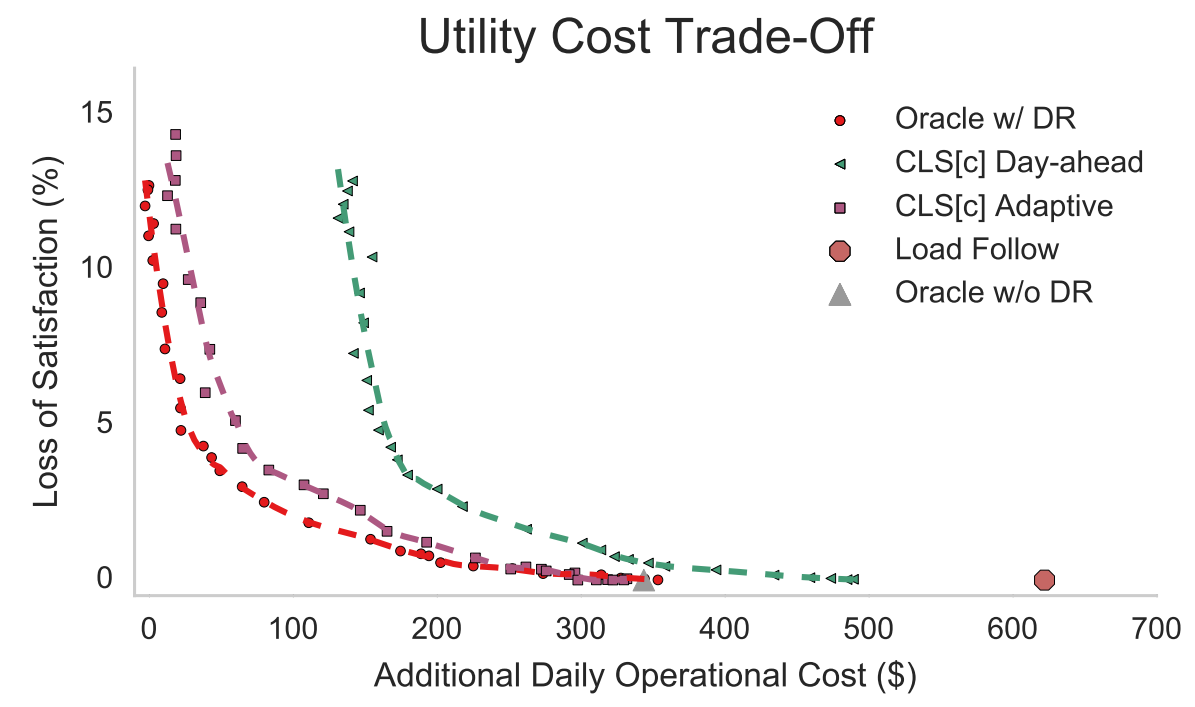

Figure 17: Loss of satisfaction and additional operational cost trade-off. The loss of satisfaction, which results from load shaping, is compared with the DR-disabled schemes (oracle without DR, load following). The additional operational cost is the extra expenditure relative to the Oracle with the most intensive DR (red dot on the top left). Each point is a 30-day average, interpolated by the dashed line for smoothness.

P1 based on CLS forecasts. The baseline model performs optimized load following, which resorts to the cheapest sources of energy available to meet the immediate loads (i.e., equivalent to a system without CHP, absorption chiller, and storage, in contrast to [37. for a rule-based operation that is price-insensitive). As the focus is on the use of existing infrastructure, fixed costs are considered sunk [68, 29].

The flexibility of DR can be utilized to optimize the operations, as depicted in Fig. 17, where each point represents a 30-day average corresponding to a particular value of utility trade-off parameter $\lambda_{u t i l}$. The DR-enabled candidates, compared with the more rigid Oracle without DR and baseline, can achieve substantial savings by balancing the utility trade-off. Even though the decision is based on forecasts, the adaptive scheme based on CLS outperforms its day-ahead counterpart, and is on a par with the DR-enabled oracle. It is at- 

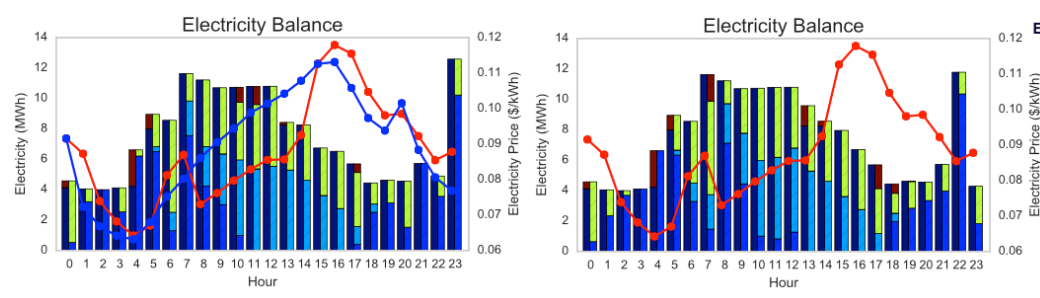

lectricity Technology

at Balance
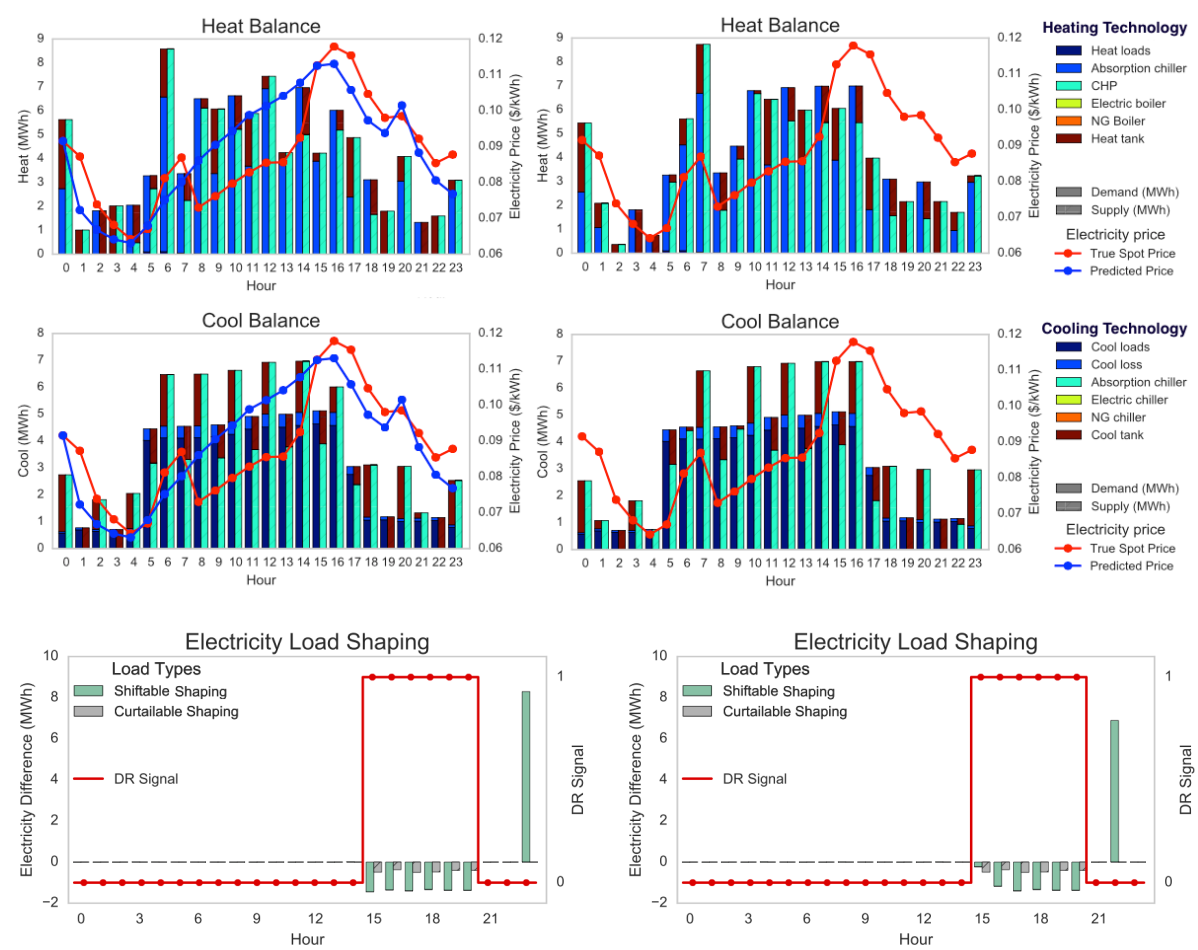

Figure 18: Power balances and load shaping for DR-enabled adaptive CLS and oracle dispatch. The predicted price for the adaptive CLS is one-hour ahead estimation, except for the first hour when the price is observed. The loads shown are after DR shaping. While the charging and discharging of electric battery is primarily to exploit the price fluctuation, thermal storage is also used to absorb the excess energy from CHP employed due to the spark spread. As the uncertainty in solar is eliminated sequentially, PV firming is not necessary.

tributed to the full utilization of PV (by dispatching in accord with the current observation of solar availability, the uncertainty for every hour is sequentially eliminated) and the dynamic adjustment of price predictions.

Importantly, peak load shedding and expenditure cutback can be made with 
Table 4: Peak load reduction and daily operational cost for each dispatch strategy in a 30day evaluation period, where the standard deviation is shown along with the average. Peak hour is defined whenever the price signal rises above the third quartile of history pool prices, triggering DR signals. In the case study, as there is sufficient supply of heat and cooling from CHPs, which are turned on during peak hours for economy, there is less need for thermal deductions compared with electricity.

\begin{tabular}{|c|c|c|c|c|c|}
\hline \multirow{2}{*}{ Method } & \multicolumn{4}{|c|}{ Peak Reduction (\%) } & \multirow{2}{*}{$\begin{array}{c}\text { Cost } \\
(\mathrm{k} \$)\end{array}$} \\
\cline { 2 - 6 } & Shiftable & Curtailable & Heat & Cool & $0(0)$ \\
\hline Load Follow & $0(0)$ & $0(0)$ & $0(0)$ & $0(0)$ & $5.7(0.5)$ \\
\hline Oracle w/o DR & $0(0)$ & $0(0)$ & $0(0)$ & $0(0)$ & $5.4(0.2)$ \\
\hline CLS[c] Day & $83.4(20.2)$ & $25.5(11.2)$ & $0.0(0.0)$ & $2.9(5.5)$ & $5.3(1.5)$ \\
\hline CLS[c] Adapt & $77.6(31.9)$ & $39.7(8.3)$ & $0.0(0.0)$ & $6.8(3.0)$ & $5.2(1.5)$ \\
\hline Oracle w/ DR & $88.7(14.2)$ & $29.4(12.4)$ & $0.0(0.0)$ & $4.0(5.6)$ & $5.1(1.5)$ \\
\hline
\end{tabular}

a pragmatic compromise of building utilities (Table 4 and Fig. 18). The adaptive strategy achieved overall $17.5 \%$ peak load reduction, which is calculated based on the actual curtailable, shiftable, and critical loads for the building clusters, and $8.8 \%$ cost savings compared to the baseline and is on par with the Oracle. Close inspection of Fig. 18 reveals that the dispatch plan by adaptive CLS resembles that of the Oracle, which is remarkable, particularly in light of the fluctuation of prices and the need to dynamically manage the shiftable and curtailable loads.

\section{Conclusion}

The MOD-DR proposed in the study engages consumers, distributed generators, and the grid in a multi-objective trade-off to derive mutual benefits, and facilitates future work on the economic analysis of real-time pricing mechanism, DR contracts, and MG valuations.

While previous work on storage capacity planning often assume deterministic renewables and electricity price for simplicity, the study indicates that the uncertainty plays a critical role in determining the operational savings. The diminishing return effect as the capacity scales up is particularly marked under 
real time pricing schemes, where the price exhibits fluctuations.

Day-ahead dispatch, based on the expectance of the electricity tariff and availability of renewables, can exploit the spark spread and the time-shifting capability of storage, thus achieving more efficient operations. The adaptive strategy, additionally, can lower the cost further by making adjustments to account for current and past information, which reduces the need for PV firming and makes the MG responsive to DR called on short notice; nevertheless, the implementation relies on robust communication and control infrastructure, which might not be readily available for some users.

From the results of the optimal dispatch plans (Figs. 9 and 18), CHPs are operated more often as the spark spread increases. On the other hand, an electric battery is charged at night when the electricity price is low, and discharged later in the day to satisfy demands, as long as the price prediction captures the peak and valley hours. Renewables can be also harvested with accurate forecasting to complement grid imports, which is conducive to lowering both the electricity bill and carbon dioxide emissions.

By inspection of the utility-cost trade-off curves (Figs. 16 and 17), as the loss of satisfaction increases, there is initially a substantial drop in the operational cost, which then diminishes as we enter into the deep DR region. The transition can be often exploited by initiating the right amount of DR on a district level to achieve considerable savings while maintaining relatively low levels of dissatisfaction.

More specifically, our main contributions and results are as follows:

- Modeling of a large-scale MG with intermittent renewables and flexible building demands under a DR scheme with real-time pricing

- Formulation of multi-objective optimization with prevailing constraints and utility trade-off, which leads to the derivation of day-ahead and adaptive dispatch strategies

700

- Corroboration of potential cost savings with accurate forecasters when dealing with the uncertainty in electricity price and renewables 
- Analysis of the benefits of electrical and thermal storage on the operational economy of MG, indicating diminishing returns under uncertain conditions

- Demonstration of the DR potential and utility-cost trade-off, showing the $17.5 \%$ peak load reduction and $8.8 \%$ cost savings with MOD-DR for a campus prototype.

One interesting aspect to explore is the dispatch at a sub-hourly resolution, where not only the shiftability of thermal loads is relevant, it is also possible to provide high frequency ancillary services to the grid. As the DR at the subhourly scale is often called on short notice, the adaptive strategy is of particular interest, which can adjust the dispatch with more agility.

Further, by leveraging the natural gas fired, electricity powered devices, and renewable sources within a MG, MOD-DR can be employed to perform fuel switching as circumstances dictate. For instance, it can effectively tackle the problem of curtailed electric energy [31, when some of the renewable energy generation must be wasted to keep real-time power balance, by optimally coordinating the load and generation sides.

Above all, the study shows the capability of the MG to serve as a flexible energy prosumer on a district level, while gaining economic efficiency and offering ancillary services to the grid. As it can be envisioned, the ongoing research of sensing, communication, and control infrastructure and deployment of MOD-DR are enablers towards an energy efficient and system-wide resilient smart power system.

\section{Acknowledgements}

This manuscript has been authored by authors at Lawrence Berkeley National Laboratory with the U.S. Department of Energy. This work is also supported by Shenzhen Institute of Building Research and the Energy Foundation. The authors would also like to thank Cecilia Han Springer and the reviewers for their constructive feedback on improving the manuscript. 
[1] A. Molderink, V. Bakker, M. G. Bosman, J. L. Hurink, G. J. Smit, Management and control of domestic smart grid technology, Smart grid, IEEE transactions on 1 (2) (2010) 109-119.

[2] H. Farhangi, The path of the smart grid, IEEE power and energy magazine 8 (1) (2010) 18-28.

[3] R. Velik, P. Nicolay, Grid-price-dependent energy management in microgrids using a modified simulated annealing triple-optimizer, Applied Energy 130 (2014) 384-395.

[4] B. P. Roberts, C. Sandberg, The role of energy storage in development of smart grids, Proceedings of the IEEE 99 (6) (2011) 1139-1144.

[5] T. Niknam, R. Azizipanah-Abarghooee, M. R. Narimani, An efficient scenario-based stochastic programming framework for multi-objective optimal micro-grid operation, Applied Energy 99 (2012) 455-470.

[6] X. He, L. Hancher, I. Azevedo, N. Keyaerts, L. Meeus, J.-M. GLACHANT, Shift, not drift: towards active demand response and beyond.

[7] A. L. Dimeas, N. D. Hatziargyriou, Operation of a multiagent system for microgrid control, IEEE Transactions on Power Systems 20 (3) (2005) $1447-1455$.

[8] Y.-H. Chen, S.-Y. Lu, Y.-R. Chang, T.-T. Lee, M.-C. Hu, Economic analysis and optimal energy management models for microgrid systems: A case study in taiwan, Applied Energy 103 (2013) 145-154.

[9] C. Marnay, G. Venkataramanan, M. Stadler, A. S. Siddiqui, R. Firestone, B. Chandran, Optimal technology selection and operation of commercialbuilding microgrids, IEEE Transactions on Power Systems 23 (3) (2008) 975-982. 
[10] H. Morais, P. Kadar, P. Faria, Z. A. Vale, H. Khodr, Optimal scheduling of a renewable micro-grid in an isolated load area using mixed-integer linear programming, Renewable Energy 35 (1) (2010) 151-156.

[11] M. Marzband, A. Sumper, A. Ruiz-Álvarez, J. L. Domínguez-García, B. Tomoiagă, Experimental evaluation of a real time energy management system for stand-alone microgrids in day-ahead markets, Applied Energy 106 (2013) 365-376.

[12] Y. Kitapbayev, J. Moriarty, P. Mancarella, Stochastic control and real options valuation of thermal storage-enabled demand response from flexible district energy systems, Applied Energy 137 (2015) 823-831.

[13] G. Díaz, B. Moreno, Valuation under uncertain energy prices and load demands of micro-chp plants supplemented by optimally switched thermal energy storage, Applied Energy 177 (2016) 553-569.

[14] G. Brusco, A. Burgio, D. Menniti, A. Pinnarelli, N. Sorrentino, Energy management system for an energy district with demand response availability, Smart Grid, IEEE Transactions on 5 (5) (2014) 2385-2393.

[15] R. Dufo-López, Optimisation of size and control of grid-connected storage under real time electricity pricing conditions, Applied Energy 140 (2015) 395-408.

775 [16] Australian Energy Market Commission, Demand response mechanism and (1) ancillary services unbundling, http://www.aemc.gov.au/Rule-Changes/ Demand-Response-Mechanism, accessed: 2016-7-10.

[17] A. Hawkes, M. Leach, Modelling high level system design and unit commitment for a microgrid, Applied energy 86 (7) (2009) 1253-1265.

780 [18] A. Baziar, A. Kavousi-Fard, Considering uncertainty in the optimal energy management of renewable micro-grids including storage devices, Renewable Energy 59 (2013) 158-166. 
[19] P. Liu, W. Feng, C. Marnay, D. Spencer, M. Jin, L. Zheng, N. Zhou, Towards the optimal development of low-carbon community energy systems, in: 2016 ACEEE Summer Study on Energy Efficiency in Buildings, ACEEE, 2016.

[20] M. Tahanan, W. van Ackooij, A. Frangioni, F. Lacalandra, Large-scale unit commitment under uncertainty, 4OR 13 (2) (2015) 115-171.

[21] A. Chaouachi, R. M. Kamel, R. Andoulsi, K. Nagasaka, Multiobjective intelligent energy management for a microgrid, IEEE Transactions on Industrial Electronics 60 (4) (2013) 1688-1699.

[22] E. Delarue, P. Van Den Bosch, W. Dhaeseleer, Effect of the accuracy of price forecasting on profit in a price based unit commitment, Electric power systems research 80 (10) (2010) 1306-1313.

[23] P. O. Kriett, M. Salani, Optimal control of a residential microgrid, Energy 42 (1) (2012) 321-330.

[24] D. Livengood, R. Larson, The energy box: Locally automated optimal control of residential electricity usage, Service Science 1 (1) (2009) 1-16.

[25] G. Celli, F. Pilo, G. Pisano, G. Soma, Optimal participation of a microgrid to the energy market with an intelligent ems, in: 2005 International Power Engineering Conference, IEEE, 2005, pp. 663-668.

[26] H. Yamin, Q. El-Dwairi, S. Shahidehpour, A new approach for gencos profit based unit commitment in day-ahead competitive electricity markets considering reserve uncertainty, International Journal of Electrical Power \& Energy Systems 29 (8) (2007) 609-616.

[27] S. A. Kazarlis, A. Bakirtzis, V. Petridis, A genetic algorithm solution to the unit commitment problem, IEEE transactions on power systems 11 (1) (1996) 83-92. 
[28] F. A. Mohamed, H. N. Koivo, Multiobjective optimization using modified game theory for online management of microgrid, European Transactions on Electrical Power 21 (1) (2011) 839-854.

[29] T. Ommen, W. B. Markussen, B. Elmegaard, Comparison of linear, mixed integer and non-linear programming methods in energy system dispatch modelling, Energy 74 (2014) 109-118.

[30] Gurobi Optimization, Inc., Gurobi optimizer reference manual version 3.0., April 2010, Houston, Texas: Gurobi Optimization.

[31] C. Li, H. Shi, Y. Cao, J. Wang, Y. Kuang, Y. Tan, J. Wei, Comprehensive review of renewable energy curtailment and avoidance: a specific example in china, Renewable and Sustainable Energy Reviews 41 (2015) 1067-1079.

[32] H. Zareipour, C. A. Canizares, K. Bhattacharya, Economic impact of electricity market price forecasting errors: a demand-side analysis, IEEE Transactions on Power Systems 25 (1) (2010) 254-262.

[33] E. Delarue, W. Dhaeseleer, Adaptive mixed-integer programming unit commitment strategy for determining the value of forecasting, Applied Energy 85 (4) (2008) 171-181.

[34] B. F. Hobbs, S. Jitprapaikulsarn, S. Konda, V. Chankong, K. A. Loparo, D. J. Maratukulam, Analysis of the value for unit commitment of improved load forecasts, IEEE Transactions on Power Systems 14 (4) (1999) 13421348.

[35] A.-H. Mohsenian-Rad, A. Leon-Garcia, Optimal residential load control with price prediction in real-time electricity pricing environments, IEEE transactions on Smart Grid 1 (2) (2010) 120-133.

[36] J.-D. Park, Y.-H. Moon, H.-J. Kook, Stochastic analysis of the uncertain hourly load demand applying to unit commitment problem, in: Power Engineering Society Summer Meeting, 2000. IEEE, Vol. 4, IEEE, 2000, pp. 2266-2271. 
[37] Y. Lu, S. Wang, Y. Sun, C. Yan, Optimal scheduling of buildings with energy generation and thermal energy storage under dynamic electricity pricing using mixed-integer nonlinear programming, Applied Energy 147 (2015) 49-58.

[38] J. M. Morales, R. Minguez, A. J. Conejo, A methodology to generate statistically dependent wind speed scenarios, Applied Energy 87 (3) (2010) 843-855.

[39] M. H. Albadi, E. El-Saadany, A summary of demand response in electricity markets, Electric power systems research 78 (11) (2008) 1989-1996.

[40] D. S. Callaway, Tapping the energy storage potential in electric loads to deliver load following and regulation, with application to wind energy, Energy Conversion and Management 50 (5) (2009) 1389-1400.

[41] A. M. Kosek, G. T. Costanzo, H. W. Bindner, O. Gehrke, An overview of demand side management control schemes for buildings in smart grids, in: Smart energy grid engineering (SEGE), 2013 IEEE international conference on, IEEE, 2013, pp. 1-9.

[42] C. De Jonghe, B. F. Hobbs, R. Belmans, Optimal generation mix with short-term demand response and wind penetration, IEEE Transactions on Power Systems 27 (2) (2012) 830-839.

[43] E. Karangelos, F. Bouffard, Towards full integration of demand-side resources in joint forward energy/reserve electricity markets, IEEE Transactions on Power Systems 27 (1) (2012) 280-289.

[44] S.-J. Kim, G. Giannakis, Scalable and robust demand response with mixedinteger constraints, Smart Grid, IEEE Transactions on 4 (4) (2013) 2089 2099.

[45] D. Patteeuw, K. Bruninx, A. Arteconi, E. Delarue, W. Dhaeseleer, L. Helsen, Integrated modeling of active demand response with electric 
heating systems coupled to thermal energy storage systems, Applied Energy 151 (2015) 306-319.

[46] A.-H. Mohsenian-Rad, V. W. Wong, J. Jatskevich, R. Schober, A. LeonGarcia, Autonomous demand-side management based on game-theoretic energy consumption scheduling for the future smart grid, Smart Grid, IEEE Transactions on 1 (3) (2010) 320-331.

[47] A. A. Moghaddam, A. Seifi, T. Niknam, Multi-operation management of a typical micro-grids using particle swarm optimization: A comparative study, Renewable and Sustainable Energy Reviews 16 (2) (2012) 12681281.

[48] Environmental and Energy Study Institute, What is district energy?, http://www.districtenergy.org/assets/pdfs/White-Papers/ What-IsDistrictEnergyEESI092311.pdf, accessed: 2016-7-10.

[49] L. A. Greening, Demand response resources: Who is responsible for implementation in a deregulated market?, Energy 35 (4) (2010) 1518-1525.

[50] C. Weber, N. Shah, Optimisation based design of a district energy system for an eco-town in the united kingdom, Energy 36 (2) (2011) 1292-1308.

[51] M. B. Blarke, Towards an intermittency-friendly energy system: Comparing electric boilers and heat pumps in distributed cogeneration, Applied Energy 91 (1) (2012) 349-365.

[52] G. Streckienè, V. Martinaitis, A. N. Andersen, J. Katz, Feasibility of chp885 plants with thermal stores in the german spot market, Applied Energy 86 (11) (2009) 2308-2316.

[53] M. Diagne, M. David, P. Lauret, J. Boland, N. Schmutz, Review of solar irradiance forecasting methods and a proposition for small-scale insular grids, Renewable and Sustainable Energy Reviews 27 (2013) 65-76. 
[59] J. Friedman, T. Hastie, R. Tibshirani, The elements of statistical learning, Vol. 1, Springer series in statistics Springer, Berlin, 2001.

[60] M. Hibon, T. Evgeniou, To combine or not to combine: selecting among forecasts and their combinations, International Journal of Forecasting 21 (1) (2005) 15-24.

[61] M. Jin, C. J. Spanos, Brief: Bayesian regression of infinite expert forecasters for single and multiple time series prediction, in: 2015 54th IEEE Conference on Decision and Control (CDC), IEEE, 2015, pp. 78-83.

[62] I. Sánchez, Adaptive combination of forecasts with application to wind 
[63] D. P. Bertsekas, D. P. Bertsekas, D. P. Bertsekas, D. P. Bertsekas, Dynamic programming and optimal control, Vol. 1, Athena Scientific Belmont, MA, 1995.

[64] A. J. Conejo, J. M. Morales, L. Baringo, Real-time demand response model, Smart Grid, IEEE Transactions on 1 (3) (2010) 236-242.

[65] S. Wilcox, W. Marion, Users manual for TMY3 data sets, 2008.

[66] J. Eyer, G. Corey, Energy storage for the electricity grid: Benefits and market potential assessment guide.

[67] B. Shen, G. Ghatikar, Z. Lei, J. Li, G. Wikler, P. Martin, The role of regulatory reforms, market changes, and technology development to make demand response a viable resource in meeting energy challenges, Applied Energy 130 (2014) 814-823.

[68] P. Sreedharan, J. Farbes, E. Cutter, C. Woo, J. Wang, Microgrid and renewable generation integration: University of California, San Diego, Applied Energy 169 (C) (2016) 709-720.

[69] H. Farhangi, Intelligent micro grid research at bcit, in: Electric Power Conference, 2008. EPEC 2008. IEEE Canada, IEEE, 2008, pp. 1-7.

[70] M. Badami, M. G. Ferrero, A. Portoraro, Dynamic parsimonious model and experimental validation of a gas microturbine at part-load conditions, Applied Thermal Engineering 75 (2015) 14-23. 


\section{APPENDIX}

Appendix .1. Thermal Balances

The heat balance is given by:

$$
\begin{aligned}
& \left(H_{t}^{\mathrm{ind}, \mathrm{pur}}-H_{t}^{\mathrm{ind}, \mathrm{sal}}\right)+\sum_{k \leq K} H_{t, k}^{\mathrm{CHP}}+\sum_{\text {tech } \in \mathcal{S}_{H, \mathrm{sp}}} H_{t}^{\mathrm{tech}}+\left(E_{t}^{\mathrm{HT}, \text { from }}-E_{t}^{\mathrm{HT}, \mathrm{sto}}\right) \\
& =H_{t}^{\mathrm{ABSchill}}+H_{t}^{\mathrm{loss}}+\sum_{b \leq B} H_{t, b}^{\mathrm{sp}}
\end{aligned}
$$

where $\mathcal{S}_{H, \mathrm{sp}}$ is comprised of electric and natural gas boilers, heat pump, and solar thermal. For cooling, the power balance is manifested as:

$$
\sum_{\text {tech } \in \mathcal{S}_{Q, \mathrm{sp}}} Q_{t}^{\mathrm{tech}}+\left(E_{t}^{\mathrm{CT}, \text { from }}-E_{t}^{\mathrm{CT}, \text { sto }}\right)=Q_{t}^{\mathrm{loss}}+\sum_{b \leq B} Q_{t, b}^{\mathrm{sp}}
$$

where $\mathcal{S}_{Q \text {,sp }}$ include electric, natural gas, and absorption chillers. We adopt a modular approach to selecting the available technologies to tailor the treatment of each case.

\section{Appendix .2. Thermal Pipeline Network}

We consider a pre-installed pipeline system for heat and cooling energy transfer, with the assumption that no thermal exchange exists among buildings, as illustrated in Fig. 3 for some prospective topologies. With the pipe segment treated as edge in the graph, the network topology can be represented using the adjacency matrix $\mathbf{A} \in\{0,1\}^{B, J}$, which assigns 1 to the entry $[\mathbf{A}]_{b, j}$ if pipe segment $j$ is employed to transport thermal energy to building $b$; for example, the adjacency matrix for topology $\mathrm{A}$ in Fig. 3 is given by:

$$
\left[\begin{array}{lllll}
1 & 0 & 0 & 0 & 1 \\
0 & 1 & 0 & 1 & 1 \\
0 & 0 & 1 & 1 & 1
\end{array}\right]
$$

We examine two cases of heat loss, as simlar for cooling. 
Length follow. Let $\mathbf{L} \in \mathbb{R}_{+}^{B}$ denote the length profile, and $\eta_{j}^{\text {len,heat }}$ be the per mile loss of heat for pipe $j$; then, the total heat transferred from the energy station, $H_{t}^{\text {src }}$, and the corresponding loss, $H_{t}^{\text {loss }}$, are given by:

$$
\begin{aligned}
H_{t}^{\mathrm{src}} & =\sum_{b \leq B} H_{t, b}^{\mathrm{sp}}+H_{t}^{\mathrm{loss}} \\
H_{t}^{\text {loss }} & =\sum_{j \leq J} \mathbf{L}_{j} \eta_{j}^{\text {len,heat }}
\end{aligned}
$$

which indicates that the total loss is the sum of individual pipe's loss proportional to the length, regardless of the actual loads on the pipe.

Load follow. With the premise that the actual loss is contingent on the loads on the pipe, we can derive:

$$
\begin{aligned}
H_{t}^{\mathrm{src}} & =\sum_{b \leq B}\left[H_{t, b}^{\mathrm{sp}} / \prod_{j \leq J}\left(\eta_{j}^{\text {ld, heat }}\right)^{[\mathbf{A}]_{b, j}}\right] \\
H_{t}^{\mathrm{loss}} & =H_{t}^{\mathrm{src}}-\sum_{b \leq B} H_{t, b}^{\mathrm{sp}}
\end{aligned}
$$

945 where the heat transfer coefficient $\eta_{j}^{\text {ld,heat }} \in(0,1)$ should, in practice, account for the length of the pipe and outside temperature effect [50].

\section{Appendix .3. Modeling of Generator Technology}

Combined Heating and Power (CHP). The MG model allows the consideration of several CHPs of diverse capacities, with each plant indexed by $k \in\{1, \ldots, K\}$ in the following. Thermal energy from burning natural gas in micro-turbines is converted to electricity,

$$
E_{t, k}^{\mathrm{CHP}}=N G_{t, k}^{\mathrm{CHP}} \cdot \eta_{k}^{\mathrm{CHP}}
$$

where the efficiency $\eta_{k}^{\mathrm{CHP}}$ is assumed constant for simplicity [13, in light of the fact that it generally degrades at lower loads [70. The remaining power, according to the heat-to-electricity ratio (HER), is recovered in part in heat recovery steam generators,

$$
H_{t, k}^{\mathrm{CHP}}=E_{t, k}^{\mathrm{CHP}} \cdot \mathrm{HER}_{k}
$$


The partial load constraint is imposed in addition to the maximum capacity to comply with desirable operating conditions [70, 13, 29]:

$$
E_{\mathrm{rat}, k}^{\mathrm{CHP}} L_{k}^{\mathrm{CHP}} X_{t, k}^{\mathrm{CHP}} \leq E_{t, k}^{\mathrm{CHP}} \leq E_{\mathrm{rat}, k}^{\mathrm{CHP}} X_{t, k}^{\mathrm{CHP}}
$$

where $L_{k}^{\mathrm{CHP}} \in(0,1)$ specifies the minimum partial load.

Electric, natural gas, and absorption chillers / boilers. For electric chiller, the process is governed by:

$$
Q_{t}^{\text {ELEChill }}=\mathrm{COP}^{\mathrm{ELEChill}} \cdot E_{t}^{\mathrm{ELEChill}}
$$

subject to the rated capacity constraints:

$$
0 \leq Q_{t}^{\text {ELEChill }} \leq Q_{\text {rat }}^{\text {ELEChill }} X_{t}^{\text {ELEChill }}
$$

The models for NG chiller and absorption chiller follow (.10) and (.11), with the replacement of electricity input $E_{t}^{\text {ELEChill }}$ in .10 to natural gas, $N G_{t}^{\mathrm{NGChill}}$, and heat, $H_{t}^{\mathrm{ABSChill}}$, suggesting the capability of lowering on-peak electricity demands and potentially operating costs when the latter resources are more economical. Whereas the absorption chiller typically has lower coefficient-ofperformance (COP) than its counterparts, it is feasible to deliver value by working in conjunction with CHP to reuse the waste heat in MG.

Electric and natural gas boilers. The technology is modeled similarly as chillers, that thermal energy is generated from sources of natural gas combustion (NG boiler) or electric resistance heating (electric boiler). The production of electric boiler is determined by

$$
H_{t}^{\text {ELEBoil }}=E_{t}^{\text {ELEBoil }} \cdot \eta^{\text {ELEBoil }},
$$

under the capacity constraints:

$$
0 \leq H_{t}^{\text {ELEBoil }} \leq H_{\text {rat }}^{\text {ELEBoil }} X_{t}^{\text {ELEBoil }}
$$

The efficiency parameter $\eta^{\text {ELEBoil }}$, which is usually high for electric boilers, is chosen as a constant to induce linearity, though the value can decrease nonlinearly at low loads [13]. The template is very much alike for natural gas boiler, 
except for the input source being natural gas, $N G_{t}^{\mathrm{NGBoil}}$, instead of electricity, $E_{t}^{\mathrm{ELEBoil}}$, which typically gives rise to higher fuel economy but also maintenance expenses.

Heat pump (HP). The HP can be engaged in either heating or cooling mode, but not both, i.e.,

$$
0 \leq X_{t}^{\mathrm{HP}, \text { heat }}+X_{t}^{\mathrm{HP}, \mathrm{cool}} \leq 1
$$

In the respective mode, the operation is described by:

$$
\begin{aligned}
& H_{t}^{\mathrm{HP}}=E_{t}^{\mathrm{HP}} \cdot \eta^{\mathrm{HP}} \cdot \mathrm{COP}_{\text {heat }}^{\mathrm{HP}}, \\
& Q_{t}^{\mathrm{HP}}=E_{t}^{\mathrm{HP}} \cdot \eta^{\mathrm{HP}} \cdot \mathrm{COP}_{\text {cool }}^{\mathrm{HP}},
\end{aligned}
$$

where $\eta^{\mathrm{HP}}$ represents the electricity energy conversion efficiency. Additionally, the rated capacity and partial loads requirements are enforced:

$$
\begin{gathered}
H_{\text {rat }}^{\mathrm{HP}} L_{\mathrm{HP}}^{\min } X_{t}^{\mathrm{HP}, \text { heat }} \leq H_{t}^{\mathrm{HP}} \leq H_{\text {rat }}^{\mathrm{HP}} X_{t}^{\mathrm{HP}, \text { heat }}, \\
Q_{\text {rat }}^{\mathrm{HP}} L_{\mathrm{HP}}^{\min } X_{t}^{\mathrm{HP}, \text { cool }} \leq Q_{t}^{\mathrm{HP}} \leq Q_{\text {rat }}^{\mathrm{HP}} X_{t}^{\mathrm{HP}, \text { cool }}
\end{gathered}
$$

with $L_{\mathrm{HP}}^{\min } \in(0,1)$. The effect of temperature difference between the source and destination, or "lift", on COP also needs to be assessed in practice.

Solar thermal and photovoltaics $(P V)$. Both technologies share the same model of operation, with the only difference in the output as either heat, $H_{t}^{\text {SolThem }}$, or electricity, $E_{t}^{\mathrm{PV}}$.

For PV, the electric power is proportional to the solar irradiation and limited by the production capacity:

$$
\begin{aligned}
& 0 \leq E_{t}^{\mathrm{PV}} \leq A^{\mathrm{PV}} \cdot \operatorname{Irr}_{t} \cdot \eta^{\mathrm{PV}} \\
& 0 \leq E_{t}^{\mathrm{PV}} \leq A^{\mathrm{PV}} \cdot e_{\mathrm{rat}}^{\mathrm{PV}}
\end{aligned}
$$

where $\eta^{\mathrm{PV}} \in(0,1)$ is the efficiency factor, $e_{\text {rat }}^{\mathrm{PV}} \in(0,1)$ is the unit out ratio that determines the rated capacity of $\mathrm{PV}$, and $\mathrm{Irr}_{t}$ is the solar irradiation and also the cause of volatility, that the PV or solar thermal cannot be dispatched at 970 will. 
Appendix .4. Modeling of Storage Technology

The heat inventory at time $t, H_{t}^{\mathrm{HT}}$, is given by:

$$
H_{t}^{\mathrm{HT}}=\left(1-\eta_{\text {decay }}^{\mathrm{HT}}\right) H_{t-1}^{\mathrm{HT}}+H_{t}^{\mathrm{HT}, \text { in }}-H_{t}^{\mathrm{HT}, \text { out }}
$$

with

$$
\begin{aligned}
H_{t}^{\mathrm{HT}, \text { in }} & =H_{t}^{\mathrm{HT}, \text { sto }} \eta_{\text {chg }}^{\mathrm{HT}} \\
H_{t}^{\mathrm{HT}, \text { from }} & =H_{t}^{\mathrm{HT}, \text { out }} \eta_{\text {dis }}^{\mathrm{HT}}
\end{aligned}
$$

where $\eta_{\text {decay }}^{\mathrm{HT}} \in(0,1)$ is the standing decay factor, and we make a distinction between $H^{H T, \text { sto }}, H^{H T, \text { out }}$ and $H^{\mathrm{HT} \text {,in }}, H^{\mathrm{HT} \text {,from }}$ as the charging / discharging energy before and after thermal losses. We also set the limits on minimum and maximum allowed rates of change [47, 5 ] as described by $\tau_{\min }^{\mathrm{HT}, \mathrm{chg}}, \tau_{\max }^{\mathrm{HT}, \mathrm{chg}}$, $\tau_{\min }^{\mathrm{HT}, \mathrm{dis}}, \tau_{\max }^{\mathrm{HT} \text {,dis }} \in(0,1)$,

$$
\begin{aligned}
H_{\text {rat }}^{\mathrm{HT}} \tau_{\text {min }}^{\mathrm{HT}, \text { chg }} X_{t}^{\mathrm{HT}, \text { chg }} & \leq H_{t}^{\mathrm{HT}, \text { in }} \leq H_{\text {rat }}^{\mathrm{HT}} \tau_{\text {max }}^{\mathrm{HT}, \text { chg }} X_{t}^{\mathrm{HT}, \text { chg }} \\
H_{\text {rat }}^{\mathrm{HT}} \tau_{\text {min }}^{\mathrm{HT}, \text { dis }} X_{t}^{\mathrm{HT}, \text { dis }} & \leq H_{t}^{\mathrm{HT}, \text { out }} \leq H_{\mathrm{rat}}^{\mathrm{HT}} \tau_{\text {max }}^{\mathrm{HT}, \text { dis }} X_{t}^{\mathrm{HT}, \text { dis }}
\end{aligned}
$$

We further disallow simultaneous charging and discharging,

$$
0 \leq X_{t}^{\mathrm{HT}, \text { chg }}+X_{t}^{\mathrm{HT}, \mathrm{dis}} \leq 1
$$

and

$$
\mathrm{SOC}_{\min }^{\mathrm{HT}} H_{\mathrm{rat}}^{\mathrm{HT}} \leq H_{t}^{\mathrm{HT}} \leq H_{\mathrm{rat}}^{\mathrm{HT}}
$$

where the lower bound specified by $\operatorname{SOC}_{\text {min }}^{\mathrm{HT}} \in(0,1)$ is commonly referred to as the spinning reserve requirement, with a typical $\mathrm{SOC}_{\min }^{\mathrm{HT}}$ of $5 \%$ [47, 5 . 